Research Article

\title{
Adaptive ADI Numerical Analysis of 2D Quenching-Type Reaction: Diffusion Equation with Convection Term
}

\author{
Xiaoliang Zhu $\mathbb{D}^{1,2}$ and Yongbin Ge iD $^{1}$ \\ ${ }^{1}$ Institute of Applied Mathematics and Mechanics, Ningxia University, Yinchuan 750021, China \\ ${ }^{2}$ School of Computer and Engineering, North Minzu University, Yinchuan 750021, China \\ Correspondence should be addressed to Yongbin Ge; gyb@nxu.edu.cn
}

Received 16 February 2020; Accepted 29 April 2020; Published 15 June 2020

Academic Editor: Ivan Giorgio

Copyright (c) 2020 Xiaoliang Zhu and Yongbin Ge. This is an open access article distributed under the Creative Commons Attribution License, which permits unrestricted use, distribution, and reproduction in any medium, provided the original work is properly cited.

\begin{abstract}
An adaptive high-order difference solution about a 2D nonlinear degenerate singular reaction-diffusion equation with a convection term is initially proposed in the paper. After the first and the second central difference operator approximating the firstorder and the second-order spatial derivative, respectively, the higher-order spatial derivatives are discretized by applying the Taylor series rule and the temporal derivative is discretized by using the Crank-Nicolson $(\mathrm{CN})$ difference scheme. An alternating direction implicit (ADI) scheme with a nonuniform grid is built in this way. Meanwhile, accuracy analysis declares the second order in time and the fourth order in space under certain conditions. Sequentially, the high-order scheme is performed on an adaptive mesh to demonstrate quenching behaviors of the singular parabolic equation and analyse the influence of combustion chamber size on quenching. The paper displays rationally that the proposed scheme is practicable for solving the $2 \mathrm{D}$ quenchingtype problem.
\end{abstract}

\section{Introduction}

Nonlinear reaction-diffusion equation with a singular or near-singular source term has been widely applied in ion conduct polarization theory [1], computational fluid dynamics [2], electromagnetism [3], material research [4], ecology [5], thermology [6, 7], and so on. Different from the linear reaction-diffusion equation, it has a chance to produce a solution with singularity. Blow-up and quenching are considered as singularity of a solution. At a limited time, the former indicates that its solution tends to infinite, and the latter indicates that its temporal derivative tends to infinite, but its solution is restricted in a certain scope $[8,9]$. Researchers often use the nonlinear singular degenerate reaction-diffusion model to simulate ignition and burning and blow-up of the fuel, which can describe burning transformation from a stable status to an unstable status in a combustion chamber and from which quenching may arise [10]. To a large extent, it is to cognize features and change laws of quenching precisely that decides the design and optimization of the combustion chamber. We pay attention to the quenching problem of the degenerative singular reaction-diffusion equation with a convection term in this paper.

Let us recall the development of the singular reactiondiffusion equation of the quenching type. Since Kawarada concluded that the time differential quotient of the solution leans toward infinity when the solution approximates to one indefinitely for this equation [1], more and more scholars have focused on nonlinear singular problems. The academic system of researching the quenching phenomena of the nonlinear reaction-diffusion equation has been gradually established, which ranges from one dimension and two dimension to three dimension [11-17]. More one-dimensional problems are investigated rather than higher-dimensional ones. However, there are still some quenching principles of the two-dimensional degenerate singular reaction-diffusion equations discovered [6-10, 12-15]. Specially, they qualitatively depicted quenching or nonquenching under certain conditions, asymptotic status 
of quenching time, the spatial definition domain size, i.e., critical length, resulting in quenching behaviors, and so on. Sheng et al. have been engaged in research in this field. Especially, Padgett and Sheng investigated the degenerate stochastic Kawarada equations from $1 \mathrm{D}$ to $2 \mathrm{D}$ and $3 \mathrm{D}$ by combining the finite difference schemes with arbitrary meshes $[11,16,17]$. The low-accuracy concepts are exploited in the above resolutions. Ge et al. firstly rendered a highorder compact difference scheme based on an adaptive mesh to study the quenching principle of the one-dimensional singular degenerate reaction-diffusion equation in 2018 [18].

Let us recall the development of the reaction-diffusion equation with a convection term of the quenching type again. Compared to the quenching equation mentioned in the prior paragraph, less attention is paid to the convectionreaction-diffusion equation with quenching singularity, especially to the $2 \mathrm{D}$ and $3 \mathrm{D}$ problems [19]. So far, it is difficult to find research on the $2 \mathrm{D}$ quenching problems. The authors in [19] discussed quenching phenomena under the conditions of different convection terms and singularity indexes: asymptotic status of quenching time, the spatial definition domain size, i.e., critical length, and so on, for the one-dimension singular equation. Sheng and Cheng studied quenching behaviors of the same type equation by using the fully adaptive difference scheme of low-order accuracy in [20]. Sheng and Khliq investigated the relationship between the critical value of the degenerative term that was not a function of spatial points and both quenching time and convergence time for the $1 \mathrm{D}$ singular degenerate problem in [21]. Zhou et al. theoretically depicted the critical length and the quenching spatial point for the convection-reactiondiffusion equation without the degenerate term [22]. Research studies above belong to the low-order difference strategy. Recently, a high-order adaptive difference scheme is firstly used to study the influence of degenerate function, convection function, nonlinear source function, and spatial definition length on quench behaviors for the one-dimensional convection-reaction-diffusion equation, respectively [23]. It provides the basis for adopting the high-precision algorithm to analyse the quenching states of the 2D convection-reaction-diffusion equation.

Depending on the previous research studies, we discover that there is an important significance for researching this two-dimensional convection-reaction-diffusion problem of the quenching type. It is well known that the finite element scheme and the finite difference scheme are popular numerical methods to solve the convection-reaction-diffusion equation [24-29]. The improved schemes based on the finite element are utilized to resolve the two-dimensional convection-reaction-diffusion problems $[24,25]$. In the past years, there have emerged many finite difference schemes for solving the two-dimensional convection-reaction-diffusion problem [26-29]. Ge and Cao built up the transformationfree high-order compact difference algorithm proposed by Kalita et al. [26] on the multigrid to solve the 2D steady convection-diffusion equation [27]. Huang proposed an ADI scheme to solve the $2 \mathrm{D}$ unsteady convection-reactiondiffusion equation, which is built by using the first and the second central difference operators to approximate the first- order and the second-order spatial derivative, respectively, and the Crank-Nicolson (CN) method to the discrete temporal derivative [28]. Li et al. represented a compact finite difference scheme of unconditional stability and the fourth order for solving the groundwater pollutant problem based on the 2D nonlinear unsteady convection-diffusion equation [29]. Of course, there exists other schemes for solving the $2 \mathrm{D}$ problem. A Fourier spectral discretization method was posted for explaining the high-dimensional fractional reaction-diffusion equations by using two separate mathematical techniques [30]. Pindza and Owolabi developed the Fourier spectral method in space and the exponential integrator scheme in time to solve the $2 \mathrm{D}$ and $3 \mathrm{D}$ fractional reaction-diffusion equations [31]. Nonlinear highdimensional convection-diffusion problems were resolved by a novel mesh-less local Petrov-Galerkin method proposed in [32]. Different from the above research studies, Owolabi and Atangana [33] studied the high-dimensional fractional reaction-diffusion systems of spatial interactions of three components' species. The dynamical system was considered to be of asymptotical stability both in local and global style by analyzing the main equation theoretically. Furthermore, a theory certifying the existence and permanence of the three species was put forward.

In general, a high-order finite difference scheme has a greater advantage comparing with other methods. An adaptive ADI difference scheme based on the two-dimensional unsteady reaction-diffusion equation with convection function is used to analyze quenching phenomena of the $2 \mathrm{D}$ combustion model through serial cases and investigate relationship between burner shape and quenching features. Due to significant practical meaning of quenching behaviors, the important quenching information is beneficial to engineering applications such as fuel burning and industry manufacturing. The compact scheme with the adaptive algorithm is employed to compute a series of important information including quenching time and quenching location. There are five parts in the paper. Section 1 introduces the theme of this study. Section 2 describes carefully the proposed scheme of high-order accuracy. Section 3 proves adaptive mesh theory. Section 4 stimulates some typical numerical samples to explore and explain quenching problems. Section 5 draws the conclusion.

\section{Problem and Difference Discretization}

2.1. Semilinear Reaction-Diffusion Equation with Convection Term. This semilinear degenerate problem model involving two spatial dimensions is regarded as equation (1) with the boundary and initialization conditions of equation (2) and (3). The solution $u(x, y, t)$ represents the temperature of the combustion chamber. $\Omega \in(0, \alpha) \times(0, \beta)$ refers to the smooth rectangle domain of the combustor, and $\partial \Omega$ is its boundary. $c_{1}(x, y)$ and $c_{2}(x, y)$ are the convection functions of $x$ and $y$, and $\sigma(x, y)=\left(x^{2}+y^{2}\right)^{q / 2}$ is the degeneracy function and $q \geq 0$. The singularity source $f(u)=1 /(1-u)^{\theta}$ is strictly increasing for $0 \leq u_{0}<1$ with $f(0)=f_{0}>0, \lim _{u \longrightarrow 1^{-}} f(u)=\infty$. The typical convection- 
reaction-diffusion equation of the quenching type is written as

$$
\sigma(x, y) u_{t}=u_{x x}+u_{y y}+c_{1}(x, y) u_{x}+c_{2}(x, y) u_{y}+f(u)
$$$$
(x, y) \in \Omega, t \in(0, T) \text {. }
$$

Its boundary conditions are

$$
u(0, y, t)=u(\alpha, y, t)=u(x, 0, t)=u(x, \beta, t)=0, \quad t \in(0, T) .
$$

Its initialization condition is

$$
u(x, y, 0)=u_{0}(x, y), \quad(x, y) \in(0, \alpha) \times(0, \beta) .
$$

With the aid of the intermediate variables $\hat{x}$ and $\hat{y}$, we replace $\hat{x}=x / \alpha$ and $\hat{y}=y / \beta$ in equation (1) which can be defined as

$$
\begin{aligned}
\sigma(\alpha \hat{x}, \beta \widehat{y}) u_{t}= & \frac{1}{\alpha^{2}} u_{\widehat{x} \widehat{x}}+\frac{1}{\beta^{2}} u_{\widehat{y y}}+\frac{c_{1}(\alpha \widehat{x}, \beta \widehat{y})}{\alpha} u_{\hat{x}}+\frac{c_{2}(\alpha \widehat{x}, \beta \widehat{y})}{\beta} u_{\widehat{y}} \\
& +f(u), \quad(\alpha \widehat{x}, \beta \widehat{y}) \in \Omega, t \in(0, T) .
\end{aligned}
$$

For the convenience of expression, we use $x$ and $y$ instead of $\hat{x}$ and $\hat{y}$. Then, the above formulation can be rearranged as

$$
\begin{aligned}
\sigma(x, y) u_{t}= & \frac{1}{\alpha^{2}} u_{x x}+\frac{1}{\beta^{2}} u_{y y}+\frac{c_{1}(x, y)}{\alpha} u_{x}+\frac{c_{2}(x, y)}{\beta} u_{y} \\
& +f(u), \quad(x, y) \in \Omega, t \in(0, T)
\end{aligned}
$$

where $\sigma(x, y)=\left(\alpha^{2} x^{2}+\beta^{2} y^{2}\right)^{q / 2}$ and $\Omega \in(0,1) \times(0,1)$. Correspondingly, the boundary conditions of equation (5) are

$$
u(0, y, t)=u(1, y, t)=u(x, 0, t)=u(x, 1, t)=0, \quad t \in(0, T) .
$$

The initialization condition of equation (5) is

$$
u(x, y, 0)=u_{0}(x, y), \quad(x, y) \in(0,1) \times(0,1) .
$$

In the physical application, we rely on equations (5) and (7) to compute $u$ and $u_{t}$. Through observing a large number of values of $u$ and $u_{t}$, we can capture the quenching moment, i.e., quenching occurs when $u$ is infinitely close to 1 and $u_{t}$ and becomes so huge that its value blows up.

2.2. Nonuniform ADI Difference Scheme. According to Taylor formula [28], the high-accuracy ADI difference scheme of equation (5) is deduced below. The steady convection-diffusion equation derived from the $1 \mathrm{D}$ unsteady convection-diffusion equation in the $x$-direction is considered as $\left(1 / \alpha^{2} \sigma(x)\right) u_{x x}+\left(c_{1}(x) / \alpha \sigma(x)\right) u_{x}=g(x)$ where $g$ is a function of $x$. By virtue of the Taylors' series expansions, we get expressions of $\left(u_{x}\right)_{k}$ and $\left(u_{x x}\right)_{k}$, including the central difference operators of the first and the second spatial derivatives, discretized on the nonuniform mesh, respectively. The two expressions are substituted into the above steady convection-diffusion equation. So, a 1D steady convection-diffusion equation dispersed at point $x_{k}$ can be written as

$$
\left[\frac{1}{\alpha^{2} \sigma_{k}}-\frac{c_{1 k}\left(x_{R}-x_{L}\right)}{2 \alpha \sigma_{k}}\right] \delta_{x}^{2} u_{k}+\frac{c_{1 k}}{\alpha \sigma_{k}} \delta_{x} u_{k}+\psi_{1 x}\left(u_{x^{3}}\right)_{k}+\psi_{2 x}\left(u_{x^{4}}\right)_{k}+\psi_{3 x}\left(u_{x^{5}}\right)_{k}=g_{k}+O\left(\frac{x_{R}^{5}+x_{L}^{5}}{x_{R}+x_{L}}\right)
$$

where

$$
\begin{aligned}
& \psi_{1 x}=-\frac{2\left(x_{R}-x_{L}\right)+\alpha c_{1 k} x_{R} x_{L}}{6 \alpha^{2} \sigma_{k}}, \\
& \psi_{2 x}=-\frac{2\left(x_{R}^{2}-x_{R} x_{L}+x_{L}^{2}\right)+\alpha c_{1 k}\left(x_{R}-x_{L}\right) x_{R} x_{L}}{24 \alpha^{2} \sigma_{k}}, \\
& \psi_{3 x}=-\frac{2\left(x_{R}^{3}+x_{R} x_{L}^{2}-x_{R}^{2} x_{L}-x_{L}^{3}\right)\left(x_{R}-x_{L}\right)-\alpha c_{1 k}\left(-x_{R}^{3} x_{L}+x_{R}^{2} x_{L}^{2}-x_{R} x_{L}^{3}\right)}{120 \alpha^{2} \sigma_{k}} .
\end{aligned}
$$

After discretizing $\left(u_{x^{3}}\right)_{k}$ and $\left(u_{x^{4}}\right)_{k}$ in the spatial direction, equation (8) is reorganized as

$$
C_{x}^{-1} P_{x} u_{k}=g_{k}+C_{x}^{-1} \psi_{3 x}\left(u_{x^{5}}\right)_{k}+O\left(\frac{x_{R}^{5}+x_{L}^{5}}{x_{R}+x_{L}}\right)
$$

where 


$$
\begin{aligned}
& P_{x}=P_{1 x} \delta_{x}+P_{2 x} \delta_{x}^{2}, \\
& P_{1 x}=\left(\frac{1}{\alpha \sigma_{k}}+P_{9 x} \delta_{x}-\alpha \psi_{2 x} \delta_{x}^{2}\right) c_{1 k}, \\
& P_{9 x}=-\alpha \psi_{1 x}+\alpha^{2} \psi_{2 x} c_{1 k}, \\
& P_{2 x}=\frac{1}{\alpha^{2} \sigma_{k}}+\left(-\frac{x_{R}-x_{L}}{2 \alpha \sigma_{k}}+P_{9 x}-2 \alpha \psi_{2 x} \delta_{x}\right) c_{1 k}, \\
& C_{x}=C_{0 x}+C_{1 x} \delta_{x}+C_{2 x} \delta_{x}^{2}, \\
& C_{0 x}=1+\left(\alpha P_{9 x} \delta_{x}-\alpha^{2} \psi_{2 x} \delta_{x}^{2}\right) \sigma_{k}, \\
& C_{1 x}=\left(\alpha P_{9 x}-2 \alpha^{2} \psi_{2 x} \delta_{x}\right) \sigma_{k}, \\
& C_{2 x}=-\alpha^{2} \psi_{2 x} \sigma_{k} .
\end{aligned}
$$

Similarly, from the one-dimensional steady convectiondiffusion equation in the $y$-direction: $\left(1 / \beta^{2} \sigma(y)\right) u_{y y}+\left(c_{2}(y) / \beta \sigma(y)\right) u_{y}=g(y)$, and the equation in the $y$-direction can be derived as

$$
C_{y}^{-1} P_{y} u_{j}=g_{j}+C_{y}^{-1} \psi_{3 y}\left(u_{y^{5}}\right)_{j}+O\left(\frac{y_{R}^{5}+y_{L}^{5}}{y_{R}+y_{L}}\right),
$$

where

$$
\begin{aligned}
P_{y} & =P_{1 y} \delta_{y}+P_{2 y} \delta_{y}^{2}, \\
P_{1 y} & =\left(\frac{1}{\beta \sigma_{j}}+P_{9 y} \delta_{y}-\beta \psi_{2 y} \delta_{y}^{2}\right) c_{2 j}, \\
P_{9 y} & =-\beta \psi_{1 y}+\beta^{2} \psi_{2 y} c_{2 j}, \\
P_{2 y}= & \frac{1}{\beta^{2} \sigma_{j}}+\left(-\frac{y_{R}-y_{L}}{2 \beta \sigma_{j}}+P_{9 y}-2 \beta \psi_{2 y} \delta_{y}\right) c_{2 j}, \\
C_{y}= & C_{0 y}+C_{1 y} \delta_{y}+C_{2 y} \delta_{y}^{2}, \\
C_{0 y}= & 1+\left(\beta P_{9 y} \delta_{y}-\beta^{2} \psi_{2 y} \delta_{y}^{2}\right) \sigma_{j}, \\
C_{1 y}= & \left(\beta P_{9 y}-2 \beta^{2} \psi_{2 y} \delta_{y}\right) \sigma_{j}, \\
C_{2 y}= & -\beta^{2} \psi_{2 y} \sigma_{j}, \\
\psi_{1 y}= & -\frac{2\left(y_{R}-y_{L}\right)+\beta y_{R} y_{L} c_{2 j}}{6 \beta^{2} \sigma_{j}}, \\
\psi_{3 y}= & -\frac{2\left(y_{R}^{3}+y_{R} y_{L}^{2}-y_{R}^{2} y_{L}-y_{L}^{3}\right)-\beta\left(-y_{R}^{3} y_{L}+y_{R}^{2} y_{L}^{2}-y_{R} y_{L}^{3}\right) c_{2 j}}{24 \beta^{2} \sigma_{j}} \\
\psi_{2 y}= & -\frac{2\left(y_{R}^{2}-y_{R} y_{L}+y_{L}^{2}\right)+\beta\left(y_{R}-y_{L}\right) y_{R} y_{L} c_{2 j}}{120},
\end{aligned}
$$

Adding the two hands of equation (10) and equation (12), we can get a $2 \mathrm{D}$ steady convection-diffusion equation:

$$
\begin{aligned}
C_{x}^{-1} P_{x} u_{k, j}+C_{y}^{-1} P_{y} u_{k, j}= & g_{k, j}+C_{x}^{-1} \psi_{3 x}\left(u_{x^{5}}\right)_{k, j}+C_{y}^{-1} \psi_{3 y}\left(u_{y^{5}}\right)_{k, j} \\
& +O\left(\frac{x_{R}^{5}+x_{L}^{5}}{x_{R}+x_{L}}\right)+O\left(\frac{y_{R}^{5}+y_{L}^{5}}{y_{R}+y_{L}}\right) .
\end{aligned}
$$

We can mark the truncation error $C_{x}^{-1} \psi_{3 x}\left(u_{x^{5}}\right)_{k, j}+C_{y}^{-1} \psi_{3 y}\left(u_{y^{5}}\right)_{k, j}+O\left(\left(x_{R}^{5}+x_{L}^{5}\right) /\left(x_{R}+x_{L}\right)\right)+$ $O\left(\left(y_{R}^{5}+y_{L}^{5}\right) /\left(y_{R}+y_{L}\right)\right)$ as $O(\Delta)$ and get

$$
\left(C_{y} P_{x}+C_{x} P_{y}\right) u_{k, j}=C_{x} C_{y} g_{k, j}+O(\Delta)
$$

Subsequently, after substituting $\left(u_{t}-(f(u) / \sigma(x, y))\right)_{k, j}$ for $g_{k, j}$ in equation (14), the situation of point $(n+1 / 2)$ for equation (15) is considered as

$$
\begin{aligned}
C_{x} C_{y}\left(u_{t}\right)_{k, j}^{n+1 / 2}= & \left(C_{y} P_{x}+C_{x} P_{y}\right) u_{k, j}^{n+1 / 2} \\
& +C_{x} C_{y}\left(\frac{f(u)}{\sigma(x, y)}\right)_{k, j}^{n+1 / 2}+O(\Delta) .
\end{aligned}
$$

The Crank-Nicolson method is used to discretize the temporal term of equation (16), and an equation of point $(n+1)$ can be formed as

$$
\begin{aligned}
{\left[C_{x} C_{y}-\frac{\tau_{n}}{2}\left(C_{y} P_{x}+C_{x} P_{y}\right)\right] u_{k, j}^{n+1}=} & {\left[C_{x} C_{y}+\frac{\tau_{n}}{2}\left(C_{y} P_{x}+C_{x} P_{y}\right)\right] u_{k, j}^{n} } \\
& +\frac{\tau_{n} C_{x} C_{y}}{2 \sigma(x, y)}\left(f_{k, j}^{n+1}+f_{k, j}^{n}\right) \\
& +O\left(\tau_{n} \Delta\right)+O\left(\tau_{n}^{3}\right),
\end{aligned}
$$

where $\tau_{n}$ is the time step and $O\left(\tau_{n} \Delta\right)+O\left(\tau_{n}^{3}\right)$ is the truncation error. When $\left(\tau_{n}^{2} / 4\right) P_{y} P_{x} u_{k, j}^{n+1}$ is added to the left side of equation (18) and $\left(\tau_{n}^{2} / 4\right) P_{y} P_{x} u_{k, j}^{n+1}$ is added to the right and the truncation error is deleted, a group of linear system is organized as

$$
\begin{aligned}
{\left[\left(C_{x}-\frac{\tau_{n}}{2} P_{x}\right)\left(C_{y}-\frac{\tau_{n}}{2} P_{y}\right)\right] u_{k, j}^{n+1}=} & {\left[\left(C_{x}+\frac{\tau_{n}}{2} P_{x}\right)\left(C_{y}+\frac{\tau_{n}}{2} P_{y}\right)\right] u_{k, j}^{n} } \\
& +\frac{\tau_{n} C_{x} C_{y}}{2 \sigma(x, y)}\left(f_{k, j}^{n+1}+f_{k, j}^{n}\right) .
\end{aligned}
$$

By virtue of the intermediate variable $u_{k, j}^{*}$, equation (18) is reorganized as

$$
\begin{aligned}
\left(C_{x}-\frac{\tau_{n}}{2} P_{x}\right) u_{k, j}^{*}= & {\left[\left(C_{x}+\frac{\tau_{n}}{2} P_{x}\right)\left(C_{y}+\frac{\tau_{n}}{2} P_{y}\right)\right] u_{k, j}^{n} } \\
& +\frac{\tau_{n} C_{x} C_{y}}{2 \sigma(x, y)}\left(f_{k, j}^{n+1}+f_{k, j}^{n}\right) \\
\left(C_{y}-\frac{\tau_{n}}{2} P_{y}\right) u_{k, j}^{n+1}= & u_{k, j}^{*} .
\end{aligned}
$$

After equation (19) is computed and arranged again, a linear system is formed as

$$
B^{* j} U^{* j}=U^{\wedge j(n)}+F^{j(n, n+1)}, \quad j=1,2,3, \ldots, J-1 .
$$


In equation (21),

$$
\begin{aligned}
& B^{* j}=\left[\begin{array}{ccccc}
b_{1,2 x}^{* j} & b_{1,3 x}^{* j} & & & \\
b_{2,1 x}^{* j} & b_{2,2 x}^{* j} & b_{2,3 x}^{* j} & & \\
& \ldots & \ldots & \ldots & \\
& & b_{K-2,1 x}^{* j} & b_{K-2,2 x}^{* j} & b_{K-2,3 x}^{* j} \\
& & & b_{K-1,1 x}^{* j} & b_{K-1,2 x}^{* j}
\end{array}\right] \text {, } \\
& U^{* j}=\left[\begin{array}{c}
u_{0, j}^{*} \\
u_{1, j}^{*} \\
u_{2, j}^{*} \\
\cdots \\
u_{k, j}^{*} \\
\cdots \\
u_{K-2, j}^{*} \\
u_{K-1, j}^{*} \\
u_{K, j}^{*}
\end{array}\right], \\
& U^{\wedge j(n)}=\left[\begin{array}{c}
u_{1, j}^{\wedge} \\
u_{2, j}^{\wedge} \\
\cdots \\
u_{k, j}^{\wedge} \\
\cdots \\
u_{K-2, j}^{\wedge} \\
u_{K-1, j}^{\wedge}
\end{array}\right], \\
& F^{j(n, n+1)}=\left[\begin{array}{c}
F_{1, j} \\
F_{2, j} \\
\cdots \\
F_{k, j} \\
\cdots \\
F_{K-2, j} \\
F_{K-1, j}
\end{array}\right] .
\end{aligned}
$$

where

$$
\begin{aligned}
& d_{k, 1 x}^{* j}=\frac{2\left(C_{2 x}+\left(\tau_{n} / 2\right) P_{2 x}\right)-x_{L}\left(C_{1 x}+\left(\tau_{n} / 2\right) P_{1 x}\right)}{x_{L}\left(x_{R}+x_{L}\right)}, \\
& d_{k, 2 x}^{* j}=\frac{-2\left(C_{2 x}+\left(\tau_{n} / 2\right) P_{2 x}\right)+C_{0 x} x_{R} x_{L}}{x_{R} x_{L}}, \\
& d_{k, 3 x}^{* j}=\frac{2\left(C_{2 x}+\left(\tau_{n} / 2\right) P_{2 x}\right)+x_{R}\left(C_{1 x}+\left(\tau_{n} / 2\right) P_{1 x}\right)}{x_{R}\left(x_{R}+x_{L}\right)}, \\
& d_{k, 1 y}^{* j}=\frac{2\left(C_{2 y}+\left(\tau_{n} / 2\right) P_{2 y}\right)-y_{L}\left(C_{1 y}+\left(\tau_{n} / 2\right) P_{1 y}\right)}{y_{L}\left(y_{R}+y_{L}\right)}, \\
& d_{k, 2 y}^{* j}=\frac{-2\left(C_{2 y}+\left(\tau_{n} / 2\right) P_{2 y}\right)+C_{0 y} y_{R} y_{L}}{y_{R} y_{L}}, \\
& d_{k, 3 y}^{* j}=\frac{2\left(C_{2 y}+\left(\tau_{n} / 2\right) P_{2 y}\right)+y_{R}\left(C_{1 y}+\left(\tau_{n} / 2\right) P_{1 y}\right)}{y_{R}\left(y_{R}+y_{L}\right)} .
\end{aligned}
$$

The element $F_{k, j}$ of $F^{j((n, n+1)}$ is

$$
\begin{aligned}
F_{k, j}= & \frac{\tau_{n}}{2 \sigma} s_{k, 1 x}^{* j}\left[s_{k, 1 y}^{* j}\left(f_{k-1, j-1}^{n}+f_{k-1, j-1}^{n+1}\right)+s_{k, 2 y}^{* j}\left(f_{k-1, j}^{n}+f_{k-1, j}^{n+1}\right)+s_{k, 3 y}^{* j}\left(f_{k-1, j+1}^{n}+f_{k-1, j+1}^{n+1}\right)\right] \\
& +\frac{\tau_{n}}{2 \sigma} s_{k, 2 x}^{* j}\left[s_{k, 1 y}^{* j}\left(f_{k, j-1}^{n}+f_{k, j-1}^{n+1}\right)+s_{k, 2 y}^{* j}\left(f_{k, j}^{n}+f_{k, j}^{n+1}\right)+s_{k, 3 y}^{* j}\left(f_{k, j+1}^{n}+f_{k, j+1}^{n+1}\right)\right] \\
& +\frac{\tau_{n}}{2 \sigma} s_{k, 3 x}^{* j}\left[s_{k, 1 y}^{* j}\left(f_{k+1, j-1}^{n}+f_{k+1, j-1}^{n+1}\right)+s_{k+1,2 y}^{* j}\left(f_{k+1, j}^{n}+f_{k+1, j}^{n+1}\right)+s_{k, 3 y}^{* j}\left(f_{k+1, j+1}^{n}+f_{k+1, j+1}^{n+1}\right)\right],
\end{aligned}
$$


where

$$
\begin{aligned}
& s_{k, 1 x}^{* j}=\frac{2 C_{2 x}-x_{L} C_{1 x}}{x_{L}\left(x_{R}+x_{L}\right)}, \\
& s_{k, 2 x}^{* j}=\frac{-2 C_{2 x}+C_{0 x} x_{R} x_{L}}{x_{R} x_{L}}, \\
& s_{k, 3 x}^{* j}=\frac{2 C_{2 x}+x_{R} C_{1 x}}{x_{R}\left(x_{R}+x_{L}\right)}, \\
& s_{k, 1 y}^{* j}=\frac{2 C_{2 y}-y_{L} C_{1 y}}{y_{L}\left(y_{R}+y_{L}\right)}, \\
& s_{k, 2 y}^{* j}=\frac{-2 C_{2 y}+C_{0 y} y_{R} y_{L}}{y_{R} y_{L}}, \\
& s_{k, 3 y}^{* j}=\frac{2 C_{2 y}+y_{R} C_{1 y}}{y_{R}\left(y_{R}+y_{L}\right)} .
\end{aligned}
$$

Similarly, from equation (20), another linear system is written as

$$
B U^{n+1}=U^{*},
$$

where

$$
B=\left[\begin{array}{ccccc}
b_{1,2 y} & b_{1,3 y} & & & \\
b_{2,1 y} & b_{2,2 y} & b_{2,3 y} & & \\
& \cdots & \ldots & \ldots & \\
& & b_{J-2,1 y} & b_{J-2,2 y} & b_{J-2,3 y} \\
& & & b_{J-1,1 y} & b_{J-1,2 y}
\end{array}\right],
$$

in which

$$
\begin{aligned}
& b_{j, 1 y}=\frac{2\left(C_{2 y}-\left(\tau_{n} / 2\right) P_{2 y}\right)-y_{L}\left(C_{1 y}-\left(\tau_{n} / 2\right) P_{1 y}\right)}{y_{L}\left(y_{R}+y_{L}\right)}, \\
& b_{j, 2 y}=\frac{-2\left(C_{2 y}-\left(\tau_{n} / 2\right) P_{2 y}\right)+C_{0 y} y_{R} y_{L}}{y_{R} y_{L}}, \\
& b_{j, 3 y}=\frac{2\left(C_{2 y}-\left(\tau_{n} / 2\right) P_{2 y}\right)+y_{R}\left(C_{1 y}-\left(\tau_{n} / 2\right) P_{1 y}\right)}{y_{R}\left(y_{R}+y_{L}\right)} .
\end{aligned}
$$

It can be observed that each $u_{k, j}^{n+1}$ on the $n+1$ th time layer is obtained, according to $u_{k, j}^{n}$ on the prior one, by computing the tridiagonal linear equations twice. We adopt the catch-up method to solve the linear equations. We rely on equation (21) to get the medial variable $u_{k, j}^{*}$, which represents the $x$-direction. Subsequently, we depend on $u_{k, j}^{*}$ and equation (28) to gain $u_{k, j}^{n+1}$, which carries information in the $y$-direction and so on, until the solutions on the last time axis are worked out. The accuracy analysis of equation (18) is performed, and its truncation error is

$$
\begin{aligned}
& O\left(\tau_{n}^{2}+\left(x_{R}^{2}+x_{L}^{2}\right)\left(x_{R}-x_{L}\right)+\left(y_{R}^{2}+y_{L}^{2}\right)\left(y_{R}-y_{L}\right)\right. \\
& \left.\quad+\frac{x_{R}^{5}+x_{L}^{5}}{x_{R}+x_{L}}+\frac{y_{R}^{5}+y_{L}^{5}}{y_{R}+y_{L}}\right) .
\end{aligned}
$$

Note that equations (19) and (20) have the second-order accuracy in time and the third- or fourth-order accuracy in space. The scheme of uniform owns spatial accuracy of the fourth order, whereas its nonuniform scheme owns spatial accuracy of the third order.

\section{Adaptive Mesh Method}

3.1. Temporal Adaptive Mesh Method. Comparing with solution $u$, the temporal derivative $u_{t}$ is more sensible. Therefore, like the $1 \mathrm{D}$ adaptive method, the $2 \mathrm{D}$ adaptive mesh method in the time direction is based on the arc-length monitor function of the temporal derivative function $u_{t}$. We can extend the $1 \mathrm{D}$ adaptive temporal grid schemes in Reference [23] to the 2D case:

$$
\frac{\tau_{n}}{\tau_{n-1}}=\frac{\max _{\substack{0<k<K \\ 0<j<J}} \sqrt{1+\left[u_{t t}\left(x_{k}, y_{j}, t_{n-1 / 2}\right)\right]^{2}}}{\max _{\substack{0<k<K \\ 0<j<J}} \sqrt{1+\left[u_{t t}\left(x_{k}, y_{j}, t_{n+1 / 2}\right)\right]^{2}}}, \quad n=1,2, \ldots
$$

After $u_{t t}$ in equation (32) is discretized through the central difference formulas to calculate the maximum ratio, the adaptive time step $\tau_{n}$ is easily obtained by equation (32) and $\tau_{n-1}$ in the prior time layer.

3.2. Spatial Adaptive Mesh Method. Similarly, we can utilize the arc-length monitor function in the space direction based on $u_{t}$ and extend from the $1 \mathrm{D}$ adaptive spatial algorithm to the $2 \mathrm{D}$ problem by relying on Reference [23]. Therefore, $W_{x}(x, t)$ representing the monitor function in the $x$-direction and $W_{y}(y, t)$ representing the monitor function in the $y$-direction can be written as

$$
\begin{aligned}
& W_{x}(x, t)=\sqrt{1+\sum_{k=0}^{K}\left[u_{t x}(x, t)\right]^{2},} \quad 0 \leq x \leq 1,0 \leq t<T, \\
& W_{y}(y, t)=\sqrt{1+\sum_{j=0}^{J}\left[u_{t y}(y, t)\right]^{2}}, \quad 0 \leq y \leq 1,0 \leq t<T .
\end{aligned}
$$

The two spatial monitor functions are positive in their definition area. $\left\{\xi_{k}\left(x_{k}, t\right)\right\}_{k=0}^{K}$ and $\left\{\zeta_{j}\left(y_{j}, t\right)\right\}_{j=0}^{j}$ represent the set of $2 \mathrm{D}$ original spatial mesh points. After an adaptive improvement is complemented, the grid in the $2 \mathrm{D}$ computational area is updated as $\left\{x_{k}\left(\xi_{k}, t\right)\right\}_{k=0}^{K}$ in the $x$-direction and $\left\{y_{j}\left(\zeta_{j}, t\right)\right\}_{j=0}^{J}$ in the $y$-direction. It is easily observed that the 1D adaptive grid scheme acts on in the $x$-direction and $y$-direction, respectively, to produce new grid points.

We take the equidistributing mesh in the $y$-direction as an example below. From [23], we know that the subareas of $W_{y}(y, t)$ in intervals of $\left[y_{j-1}(t), y_{j}(t)\right](j=1,2, \ldots, J)$ are equal. Obviously, when $W_{y} \equiv 1$, the scope $[0,1]$ is uniform. It can be expressed as 


$$
\int_{y_{0}}^{y_{1}} W_{y}(y, t) \mathrm{d} y=\int_{y_{1}}^{y_{2}} W_{y}(y, t) \mathrm{d} y=\cdots=\int_{y_{J-1}}^{y_{J}} W_{y}(y, t) d y=\frac{1}{J} \int_{0}^{1} W_{y}(y, t) \mathrm{d} y=\varsigma_{y}(t),
$$

where $\varsigma_{y}(t)$ is the average arc-length. Equation (35) is equal

to

$$
\int_{y_{j-1}}^{y_{j}} W_{y}(y, t) \mathrm{d} y=\int_{\zeta_{j-1}}^{\zeta_{j}} W_{y}(y(\zeta, t), t) y^{\prime}(\zeta, t) \mathrm{d} \zeta, \quad j=1,2, \ldots, J .
$$

After the second derivation of the aforementioned formula, the following equation can be obtained:

$$
\frac{\partial}{\partial \zeta}\left(W_{y}\left(y_{j}^{(m)}, t\right) \frac{\partial}{\partial \zeta}\left(y_{j}^{(m+1)}\right)\right)=0
$$

where $m$ represents the iterative number, $\left\{y_{j}^{(m)}\right\}_{j=0}^{J}$ represents the spatial grid node set before iteration, and $\left\{y_{j}^{(m+1)}\right\}_{j=0}^{J}$ represents the space grid node set after iteration. Equation (37) can be solved by the difference scheme:

$$
W_{y}\left(y_{j+1 / 2}^{(m)}, \tau_{n}\right)\left(y_{j+1}^{(m+1)}-y_{j}^{(m+1)}\right)-W_{y}\left(y_{j-1 / 2}^{(m)}, \tau_{n}\right)\left(y_{j}^{(m+1)}-y_{j-1}^{(m+1)}\right)=0, \quad j=1,2, \ldots, J-1, n \geq 0
$$

which approximates to the former. Similarly, the semiimplicit scheme in the $x$-direction is defined as

$$
W_{x}\left(x_{k+1 / 2}^{(m)}, \tau_{n}\right)\left(x_{k+1}^{(m+1)}-x_{k}^{(m+1)}\right)-W_{x}\left(x_{k-1 / 2}^{(m)}, \tau_{n}\right)\left(x_{k}^{(m+1)}-x_{k-1}^{(m+1)}\right)=0, \quad k=1,2, \ldots, K-1, n \geq 0 .
$$

3.3. Iterative Adaption Method. The adaptive iterative procedure of the presented method is carried forward as follows. Firstly, the spatial monitor functions $W_{x}(x, t)$ and $W_{y}(y, t)$ can be obtained through equations (33) and (34) based on the grids $\left\{x_{k}^{(m)}\right\}_{k=0}^{K}$ and $\left\{y_{j}^{(m)}\right\}_{j=0}^{J}$ in the $n$th time layer. Secondly, depending on equations (38) and (39), we can gain $\left\{x_{k}^{(m+1)}\right\}_{k=0}^{K}$ derived from $\left\{x_{k}^{(m)}\right\}_{k=0}^{K}$ in the $x$-direction and $\left\{y_{j}^{(m+1)}\right\}_{j=0}^{J}$ derived from $\left\{y_{j}^{(m)}\right\}_{j=0}^{J}$ in the $y$-direction. The new points substitute for the old points iteratively when $\sum_{k=1}^{K-1}\left\|x_{k}^{(m+1)}-x_{k}^{(m)}\right\|<e r$ and $\sum_{j=1}^{J-1}\left\|y_{j}^{(m+1)}-y_{j}^{(m)}\right\|<e r$ are reached for $0<e r \leq 1 . m_{\max }$ is the terminal value of $m+1$. Thirdly, from the value built on the last grid point sets $\left\{x_{k}^{\left(m_{\max }\right)}\right\}_{k=0}^{K}$ and $\left\{y_{j}^{\left(m_{\max }\right)}\right\}_{j=0}^{J},\left\{u_{k, j}^{n}\right\}_{k=1, j=1}^{K-1, J-1}$ is computed by using the method of area ratio. Thus, we can get $\left\{u_{k, j}^{n+1}\right\}_{k=1, j=1}^{k-1, J-1}$ in the $n+1$ th time layer depending on equation (18). Lastly, repeat the aforementioned produce till quenching occurs or the solution converges to a steady solution.

\section{Numerical Illustration}

4.1. Common Example. It is not a quenching case. It is just for demonstrating the accuracy and convergence of the proposed difference scheme. We have solved the following unsteady convection-diffusion equation with coefficient whose exact solution is offered:

$$
\begin{gathered}
p u_{t}=u_{x x}+u_{y y}-p u_{x}-p u_{y}, \quad(x, y) \in[0,1] \times[0,1], t \geq 0, \\
u(x, y, t)=\frac{e^{p x}-1}{e^{p}-1}+\frac{e^{p y}-1}{e^{p}-1}+\sum_{q=1}^{\infty} \frac{(-1)^{q} q \pi}{(q \pi)^{2}+p^{2} / 4} e^{p(x-1) / 2+p(y-1) / 2} \sin (q \pi x+q \pi y) e^{-\left[(q \pi)^{2} / p+p / 4\right] t} .
\end{gathered}
$$


The initial and boundary value conditions are derived from the exact solution. There are four metrics for the data test, which are maximum error, average error, CPU time, and convergence rate. We adopt these parameters $T=0.5, \tau_{n}=0.01$, and $p=100$ to fulfill the two difference schemes: the proposed scheme based on the uniform mesh and the proposed scheme based on the nonuniform mesh. $\left\{k / K+\left(\lambda_{x} / \pi\right) \sin (\pi k / K)\right\}_{k=0}^{K}$ and $\left\{j / J+\left(\lambda_{y} / \pi\right) \sin (\pi j / J)\right\}_{j=0}^{J}$ represent the nonuniform grid, where $K$ and $J$ point to intervals of the spatial direction and $K=J$, whereas $\lambda_{x}$ and $\lambda_{y}$ point to the mesh transformation coefficient and $\lambda_{x}=\lambda_{y}$.

Table 1 offers these measuring data. It displays the accuracies and running time of our scheme (uniform and nonuniform). We select five spatial intervals: $16,32,64,128$, and 256 as test cases, which are given to each of the above two meshes (i.e., uniform and nonuniform). For the nonuniform mesh, the different values of $\lambda_{x}$ impact these precision criteria. In the paper, $\lambda_{x}$ takes 0.7 . The maximum errors of the first scheme are higher than those of the second one, and the average errors of the latter are superior to those of the former. Furthermore, the larger the number of spatial intervals is, the higher the accuracies of the scheme based on the nonuniform grid are. Its accuracies arrive at $1.3364 \times 10^{-7}$ in the maximum error and $6.4492 \times 10^{-9}$ in the average error when $K=J=256$. In terms of computing time of the two schemes whose measurement unit is millisecond, the first scheme is lower than the second one. Obviously, by comparing with the uniform mesh, the difference scheme based on the nonuniform mesh spends more time to run. Lastly, the conv. rate values are observed. The tendency of the conv. rate from the former scheme is similar to that from the latter scheme. We take the latter as the example. The four values are positive, which means the accuracy increases as $K$ rises when $K \in[16,256]$. The conv. rate value is maximum when $K$ transfers from 16 to 32 , which means the accuracy promotes greatly in the stage. The latter three values are close, which means the accuracy promotes gently in the three stages.

4.2. Quenching Case without Convection Term. This section will describe a quenching status without the convection term, i.e., $c_{1}(x, y)=0$ and $c_{2}(x, y)=0$. The mathematical model can be expressed as

$$
\begin{aligned}
\left(\alpha^{2} x^{2}+\beta^{2} y^{2}\right)^{q / 2} u_{t}= & \frac{1}{\alpha^{2}} u_{x x}+\frac{1}{\beta^{2}} u_{y y}+\frac{1}{(1-u)^{\theta}}, \\
& (x, y) \in(0,1) \times(0,1), t \in(0, T) .
\end{aligned}
$$

Its initial and boundary conditions are just as in equations (6) and (7).

After equations (19) and (20) are used to approximate equation (41), we can get some quenching behaviors. For the 2D nonlinear degeneration singularity equation without the convection term, we demonstrate the quenching case of $q=$ 0 that has been described in $[13,14]$. In Table 2 and Figure 1, $q=0, a=\beta=\sqrt{10}$, and $\theta=1$ which are the same for the three approaches, but the initial space step $h_{0}$ and the initial temporal step $\tau_{0}$ are different among them, where we take $h_{0}=1 / 50$ and $\tau_{0}=4.166677 \times 10^{-3}$. In Table 2 , the five parameters represent the same senses, in which $\left(x_{\max }, y_{\max }\right)$ represents the point of quenching location, $t_{\max }$ represents the quenching time, max $u$ represents the maximum temperature value immediately before quenching happens, and $\max u_{t}$ means the maximum variation of temperature with respect to time immediately before quenching happens. From Table 2, we can see that the quenching location $(0.5,0.5)$ of the referred scheme is identical with that of the scheme in [14], and $t_{\max }=0.5958348109999995$ and $\max u=0.998562875689732$ of the first one are close to those of the last two. Obviously, $\max u_{t}$ is different among the various methods. From Figure 1, it is found that $u \longrightarrow 1^{-}$and $u_{t} \longrightarrow 690^{-}$at the location $\left(x_{\max }, y_{\max }\right)=(0.5,0.5)$ and the quenching time $t_{\max }=0.5958348109999995$.

4.3. Quenching Case with Convection Term $b /(1+\alpha x+\beta y)$. The standard 2D degenerate semilinear quenching problem with the initial boundary condition lists in equations (5)-(7) is extended from the equation in one-space dimension [23]. The first convection term $b /(1+\alpha x+\beta y)$ can be written as two functions $c_{1}(x, y)=b_{x} /(1+\alpha x+\beta y)$ and $c_{2}(x, y)=b_{y} /(1+\alpha x+\beta y)$, where $b_{x}$ and $b_{y}$ take the constants. The four groups of variables: $q, b_{x}$ and $b_{y}, \alpha$ and $\beta$, and $\theta$ in equations (5)-(7) affect the quenching characteristics of the $2 \mathrm{D}$ problem, whose meaning has been stated in Section 2. There follows a special case for $b /(1+\alpha x+\beta y)$. The initial time step is considered as $\tau_{0}=0.0003$, and the initial space step is considered as $h_{0}=1 / 55$, in which the $x$-direction is identical with the $y$-direction. The four groups of parameters are configured as $q=0.5, \alpha=\beta=\sqrt{200}, b_{x}=$ $b_{y}=10$, and $\theta=1$ which is defined as Case 7.0. This will lead to a quenching phenomenon. Quenching location $\left(x_{7}^{*}, y_{7}^{*}\right)$ is (0.03636363636363636, 0.03636363636363636); the quenching time $T_{7}^{*}$ is $4.487776579638927 ; \max _{x, y} u$ is $0.9992409498317957 ; \max _{x, y}\left(u_{t}\right)$ is 1539.153786381783 . When $t$ is 4.486155872847072 , the temporal step starts to become smaller and smaller until quenching occurs. We choose six rows of representative data given in Table 3 which poins to features relative to $x, y, t, u$, and $u_{t}$ before quenching occurs of Case 7.0. $\max _{x, y} u$ and $\max _{x, y}\left(u_{t}\right)$ refer to maximal $u$ and maximal $u_{t}$ in each time layer before quenching occurs; $x$ and $y$ refer to the coordinate point of synchronously producing $\max _{x, y} u$ and $\max _{x, y}\left(u_{t}\right)$ in each time layer before quenching occurs; $t$ refers to the moment at which each time layer is located before quenching occurs. $(x, y)$ first appears at the point $\left(x_{7}^{*}, y_{7}^{*}\right)$ when $t$ is 4.487088240486234 which is the first row in Table 3 and maintains at this point until quenching occurs. When $t$ is $T_{7}^{*}$ which is the immediate time before quenching happens, we can obtain important characteristics. When $t$ is 4.487777138788559, $\max _{x, y}\left(u_{t}\right)$ blows up and reaches $1.051076326841211 \times 10^{9}$.

Figures 2-7 provide seven groups of graphs to illustrate more particular quenching statuses. Figure 2 shows the $2 \mathrm{D}$ 
TABLE 1: The parameter values for common example.

\begin{tabular}{|c|c|c|c|c|c|}
\hline Difference schemes & $K(J)$ & Max. error & Aver. error & CPU time & Conv. rate \\
\hline \multirow{5}{*}{ The proposed scheme (uniform) } & 16 & $2.4859 \times 10^{-1}$ & $1.7858 \times 10^{-2}$ & 47 & - \\
\hline & 32 & $5.7032 \times 10^{-2}$ & $1.9148 \times 10^{-3}$ & 172 & 5.0033 \\
\hline & 64 & $5.5382 \times 10^{-3}$ & $1.4565 \times 10^{-4}$ & 702 & 3.7166 \\
\hline & 128 & $3.1469 \times 10^{-4}$ & $9.6850 \times 10^{-6}$ & 2948 & 3.9106 \\
\hline & 256 & $1.9710 \times 10^{-5}$ & $6.1743 \times 10^{-7}$ & 11794 & 3.9714 \\
\hline \multirow{5}{*}{ The proposed scheme (nonuniform) } & 16 & $8.1898 \times 10^{-3}$ & $8.7725 \times 10^{-4}$ & 358 & - \\
\hline & 32 & $2.9724 \times 10^{-4}$ & $2.9996 \times 10^{-5}$ & 2855 & 7.9861 \\
\hline & 64 & $1.5573 \times 10^{-5}$ & $1.6521 \times 10^{-6}$ & 14914 & 4.1824 \\
\hline & 128 & $9.3004 \times 10^{-7}$ & $1.0224 \times 10^{-7}$ & 35006 & 4.0143 \\
\hline & 256 & $1.3364 \times 10^{-7}$ & $6.4492 \times 10^{-9}$ & 165969 & 3.9867 \\
\hline
\end{tabular}

TABLE 2: Quenching performances of different schemes for $q=0, a=\beta=\sqrt{10}$, and $\theta=1$ without the convection term.

\begin{tabular}{lccccc}
\hline Schemes & $t_{\max }$ & $x_{\max }$ & $y_{\max }$ & $\max u$ & $\max u_{t}$ \\
\hline The proposed scheme & 0.5958348109999995 & 0.500 & 0.500 & 0.998562875689732 & 689.2724781649539 \\
The scheme in [13] & 0.58712499993751 & - & - & 0.990432 & 148.887767 \\
The scheme in [14] & 0.587554 & 0.500 & 0.500 & 0.999263 & 1249.917563 \\
\hline
\end{tabular}

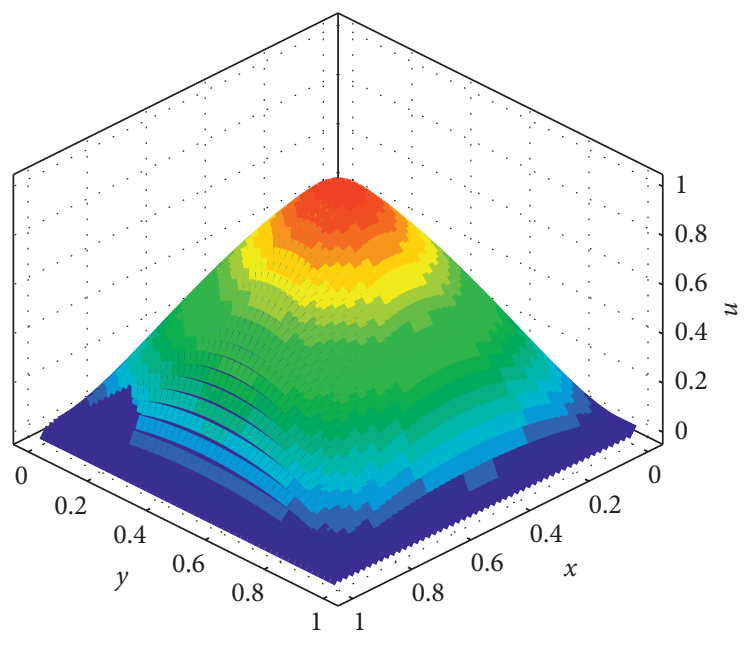

(a)

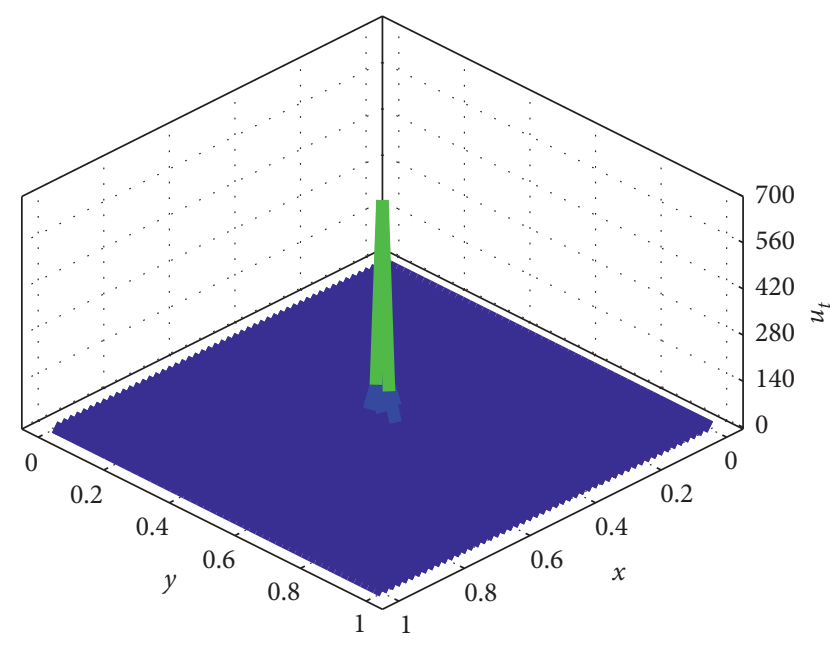

(b)

Figure 1: (a) The 3D plots of $u$ immediately before quenching occurs and (b) the 3D plots of $u_{t}$ immediately before quenching occurs. The parameters are $q=0, a=\beta=\sqrt{10}$, and $\theta=1$ without the convection term.

TABLE 3: Time, location, $\max _{x, y} u$, and $\max _{x, y}\left(u_{t}\right)$ in the six temporal layers which contain quenching occurrence for Case 7.0.

\begin{tabular}{lccrr}
\hline$t$ & $x$ & $y$ & $\max _{x, y} u$ & $\max _{x, y}\left(u_{t}\right)$ \\
\hline 4.487088240486234 & 0.03636363636363636 & 0.03636363636363636 & 0.9794485698289615 & 52.18443133976322 \\
4.487489880800603 & 0.03636363636363636 & 0.03636363636363636 & 0.9854614620545389 & 75.52899759027348 \\
4.487653824098646 & 0.03636363636363636 & 0.03636363636363636 & 0.9897483333199262 & 109.0744740434431 \\
4.487770702255042 & 0.03636363636363636 & 0.03636363636363636 & 0.9972318816440022 & 418.0023774450405 \\
4.487776579638927 & 0.03636363636363636 & 0.03636363636363636 & 0.9992409498317957 & 1539.153786381783 \\
4.487777138788559 & - & - & - & $1.051076326841211 \times 10^{9}$ \\
\hline
\end{tabular}

plots of $\max _{x, y} u$ and $\max _{x, y}\left(u_{t}\right)$ changing as $t$ rises from the initial moment until quenching occurs. Figure 2(a) corresponds to $u$, and Figure 2(b) corresponds to $u_{t}$. Figure 3 sketches the $2 \mathrm{D}$ contours of $u$ and $u_{t}$ regarding $x$ immediately before quenching happens. Figure 3(a) corresponds to $u$, and Figure 3 (b) corresponds to $u_{t}$. Figure 4 draws the
2D outlines of $u$ and $u_{t}$ regarding $y$ immediately before quenching happens. Figure 4(a) corresponds to $u$, and Figure 4(b) corresponds to $u_{t}$. The three 2D plots in Figure 5 describe the adaptive mechanism before quenching occurs. The first one reflects spatial adaptation, and the last two reflect temporal adaptation. Figure 5(a) describes the trend 


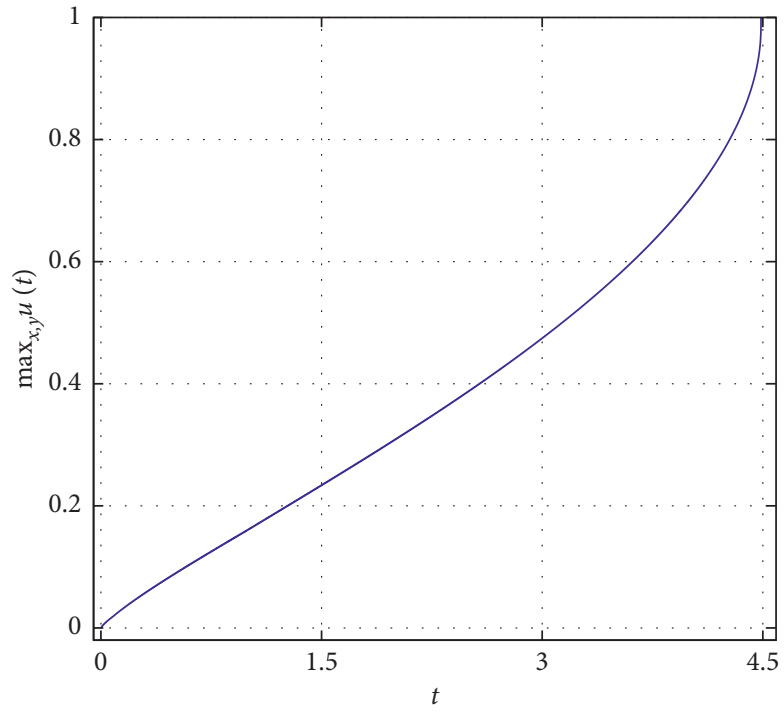

(a)

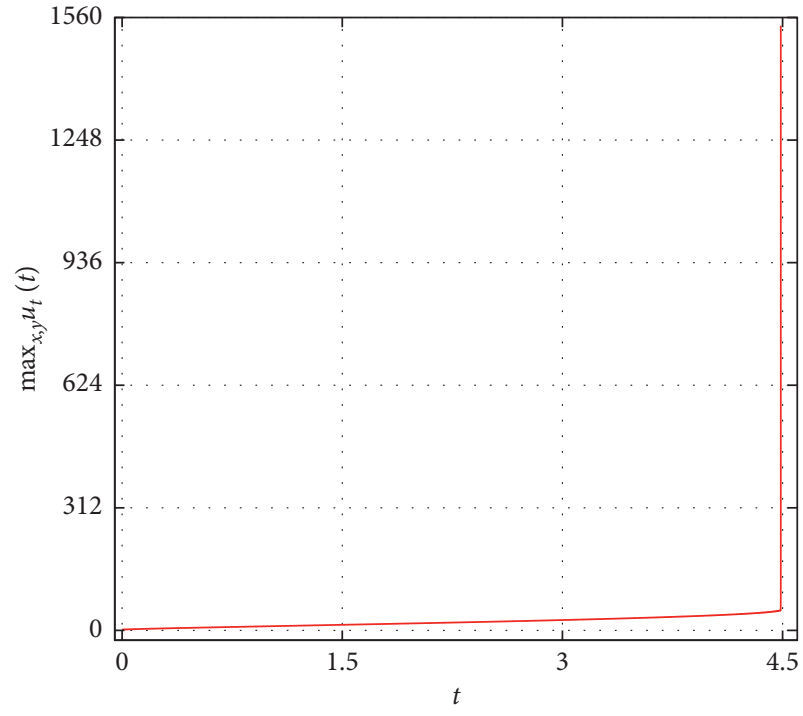

(b)

FIGURE 2: The graphs of (a) $\max _{x, y} u$ as $t$ increases and (b) $\max _{x, y}\left(u_{t}\right)$ as $t$ increases from the initial moment to the occurrence of quenching behavior for the convection term $b /(1+\alpha x+\beta y)$.

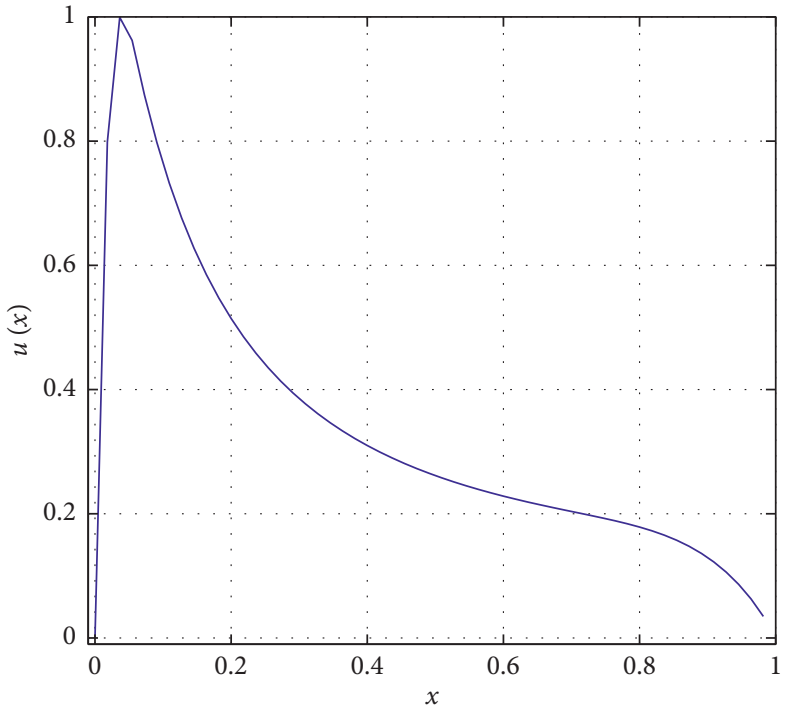

(a)

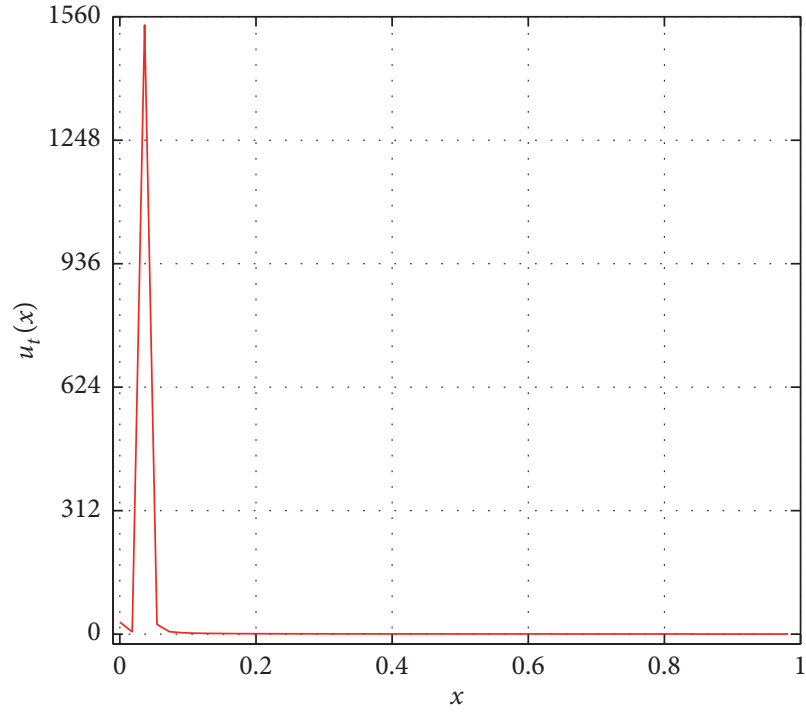

(b)

Figure 3: The graphs of (a) $u$ as $x$ increases and (b) $u_{t}$ as $x$ increases when $t$ is $T_{7}^{*}$ for the convection term $b /(1+\alpha x+\beta y)$.

of the temporal step over time. To distinguish more clearly the variation, we intercept the curves of the period from 4.470000000001407 to 4.487777138788559 . The red sign on the blue curve in Figure 5(a) denotes the initial temporal adaptive moment (i.e., 4.486155872847072). The temporal step is uniform before the adaptive moment but becomes nonuniform when $t$ varies from 4.486155872847072 to 4.487777138788559. The green sign on the blue curve in Figure 5(a) denotes the initial moment (i.e., 4.487088240486234) of producing the quenching location $\left(x_{7}^{*}, y_{7}^{*}\right)$. The green lines in Figures 5(b) and 5(c) describe the uniform spatial steps in the $x$-direction and the $y$-direction, respectively. The spatial adaptation occurs at 4.487777138788559 after which the spatial step becomes nonuniform. The red curves with blue spots in Figures 5(b) and 5(c) describe, respectively, the nonuniform spatial steps in the $x$-direction and the $y$-direction at $T_{7}^{*}$. Although the two curves are very similar, there is still subtle difference between their data. Figures 6-7 provide the 3D surfaces of $u$ and $u_{t}$ with respect to spatial variables $x$ and $y$ at certain special time. Figure 6(a) records the 3D surfaces about $x, y$, and $u$, and Figure $6(\mathrm{~b})$ records the $3 \mathrm{D}$ surfaces about $x, y$, 


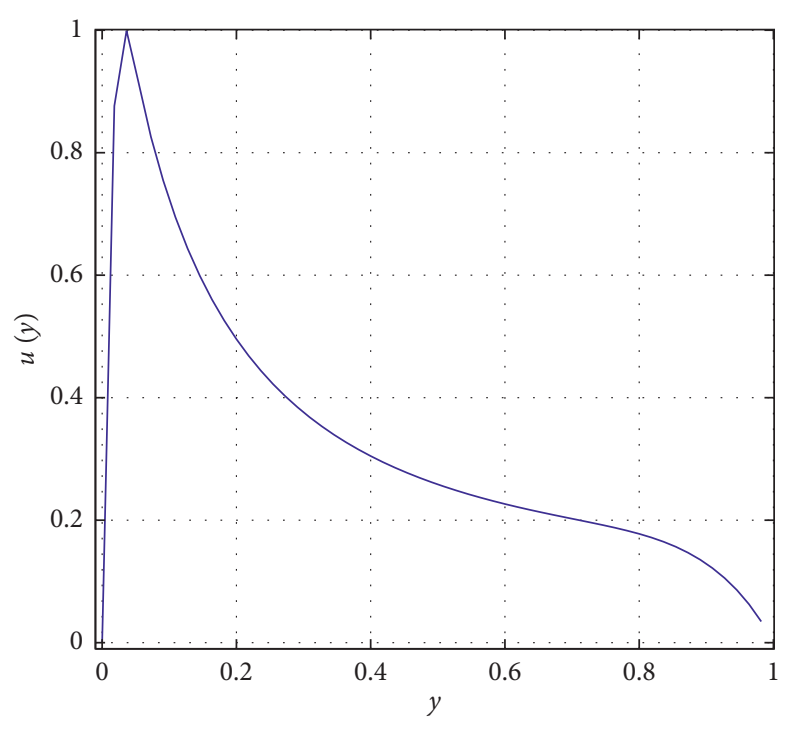

(a)

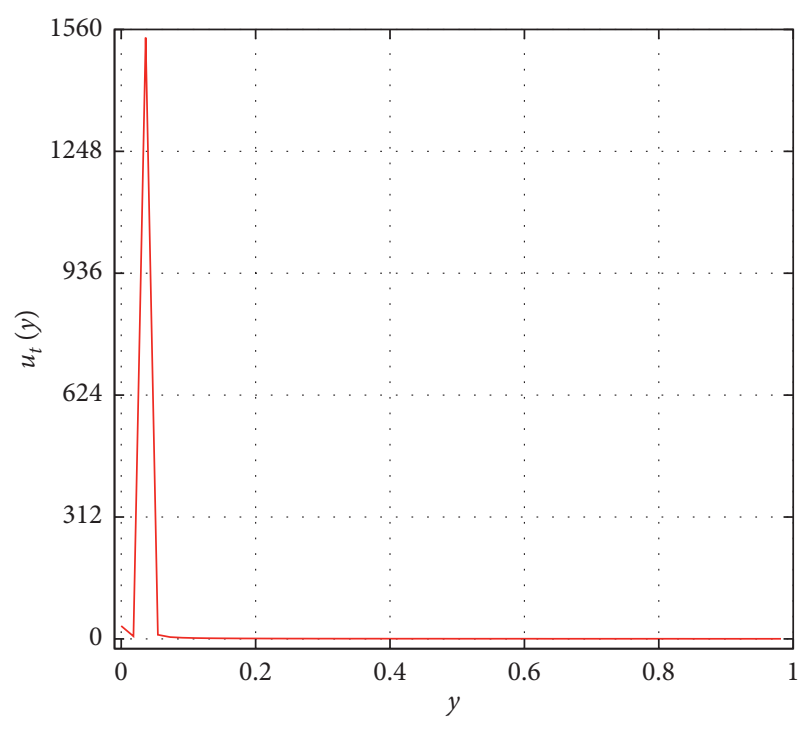

(b)

Figure 4: The graphs of (a) $u$ as $y$ increases and (b) $u_{t}$ as $y$ increases when $t$ is $T_{7}^{*}$ for the convection term $b /(1+\alpha x+\beta y)$.

and $u_{t}$ when $t=4.487770702255042$. Figure 7(a) records the 3D surfaces about $x, y$, and $u$, and Figure 7(b) records the 3D surfaces about $x, y$, and $u_{t}$ when $T_{7}^{*}$.

4.4. Quenching Case with Convection Term $b /(\alpha x+\beta y)$. The second convection term $b /(\alpha x+\beta y)$ can be written as the two convection functions: $c_{1}(x, y)=b_{x} /(\alpha x+\beta y)$ and $c_{2}(x, y)=b_{y} /(\alpha x+\beta y)$, where $b_{x}$ and $b_{y}$ take the constants. Comparing with the first convection term $b /(1+\alpha x+\beta y)$, it is hard to conduct quenching behavior for the second convection term $b /(\alpha x+\beta y)$. We make a following configuration to gain a quenching status. The initial $x$-step ( $y$-step) is $h_{0}=1 / 40$, and the initial $t$-step is $\tau_{0}=0.0005$. The four groups of important parameters are set as $q=$ $1.1, \alpha=\beta=20, b_{x}=b_{y}=3$, and $\theta=1$ which is defined as Case 8.0. The parameter statuses of the case are recorded in Table 4 . For the case, the initial time of producing the quenching location is earlier than the temporal adaptive time. The former time is 5.596499999999618 , which is the first row in Table 4, the latter one is 5.603982012140827, which is the fifth row in Table 4 , and the quenching location $\left(x_{8}^{*}, y_{8}^{*}\right)$ is $(0.025,0.025)$. Moreover, it is so transient from the front time of quenching location appearing to the terminative time of quenching location appearing that there are 18 time layers between the two moments. The latter quenching time is $\left(T_{8}^{*}=5.604262757909247\right), \max _{x, y} u$ is 0.9835341519876587 , and $\max _{x, y}\left(u_{t}\right)$ is 73.15674241637485 immediately before quenching occurs, which is the sixth row in Table 4. $\max _{x, y}\left(u_{t}\right)$ gets the value $6.637517818582074 \times 10^{11}$ and blows up when $t$ is 5.604272047535358 , which is the last row in Table 4.

This paragraph supplies five sets of figures (i.e., Figures 8-12) to more vividly depict the quenching information of the case. The 2D curves in Figure 8 describe the relationship between $\max _{x, y} u$ and $t$ and the relationship between $\max _{x, y}\left(u_{t}\right)$ and $t$. The $2 \mathrm{D}$ curves in Figure 9 describe the relationship between $u$ and $x$ and the relationship between $u_{t}$ and $x$. The 2D curves in Figure 10 describe the relationship between $u$ and $y$ and the relationship between $u_{t}$ and $y$. In Figures 8-10, (a) corresponds to $u$ and (b) corresponds to $u_{t}$. Figure 11(a) draws the tendency of the temporal step varying with temporal variable $t$; Figure 11(b) draws the tendency of the initial spatial step and the adaptive step varying with spatial variable $x$; and Figure 11(c) draws the tendency of the initial spatial step and the adaptive step varying with spatial variable $y$. For Figure 11(a), to distinguish more clearly the variation, we intercept the curves of the period from 5.580499999999627 to 5.604272047535358; and the red spot on the blue curve expresses the earliest temporal adaptive time (i.e., 5.603982012140827). $\max _{x, y}\left(u_{t}\right)$ in the time step becomes $1.185615675885128 \times 10^{12}$ when $t$ is 5.603982012140827 . The temporal step is uniform before the adaptive moment but becomes nonuniform since then. The green spot on the blue curve expresses the initial moment of producing the quenching location $\left(x_{8}^{*}, y_{8}^{*}\right)$, which is 5.604262757909247 . In Figures 11(b) and 11(c), the green lines indicate the uniform initial spatial steps while the red curves with blue signs indicate the nonuniform adaptive spatial steps. Lastly, we provide two 3D surfaces in Figure 12 to state quenching statuses of $u$ and $u_{t}$ when $t=T_{8}^{*}$. Figure 12(a) denotes the relationship between $u, x$, and $y$, and Figure 12(b) denotes the relationship between $u_{t}, x$, and $y$. Figure 12(a) records the 3D surfaces about $x, y$, and $u$, and Figure 12(b) records the $3 \mathrm{D}$ surfaces about $x, y$, and $u_{t}$ when $t=T_{8}^{*}$. There is a narrowband projecting the rear plane of the $3 \mathrm{D}$ axis box in Figure 12(b). This is because some larger values of $\max _{x, y}\left(u_{t}\right)$ appear at the positions, in which the $x$-axis and the $y$-axis are simultaneously near the origin of the coordinates. From the data source of the 3D surface, we can observe some larger $\max _{x, y}\left(u_{t}\right)$ varying from 1 to 27 , 


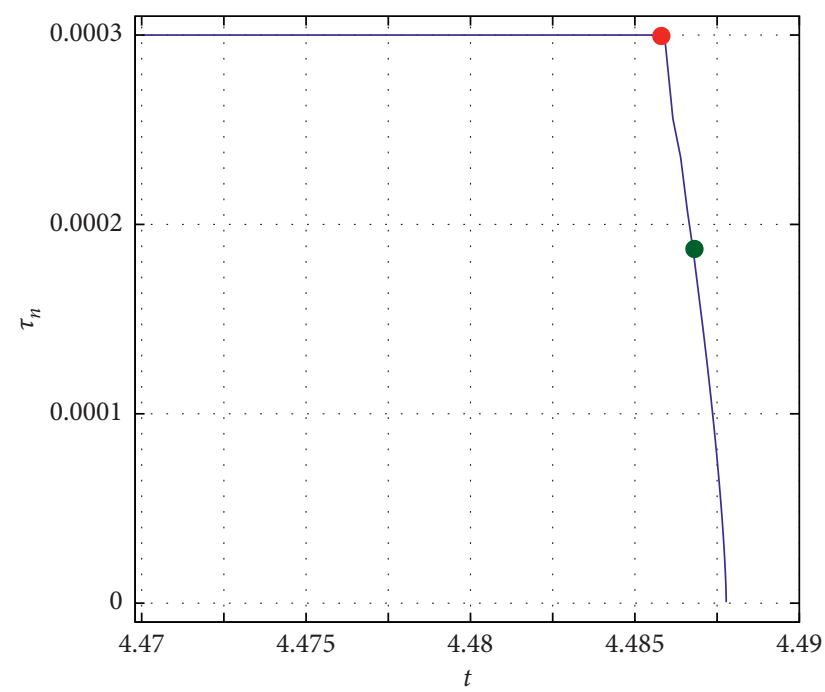

(a)

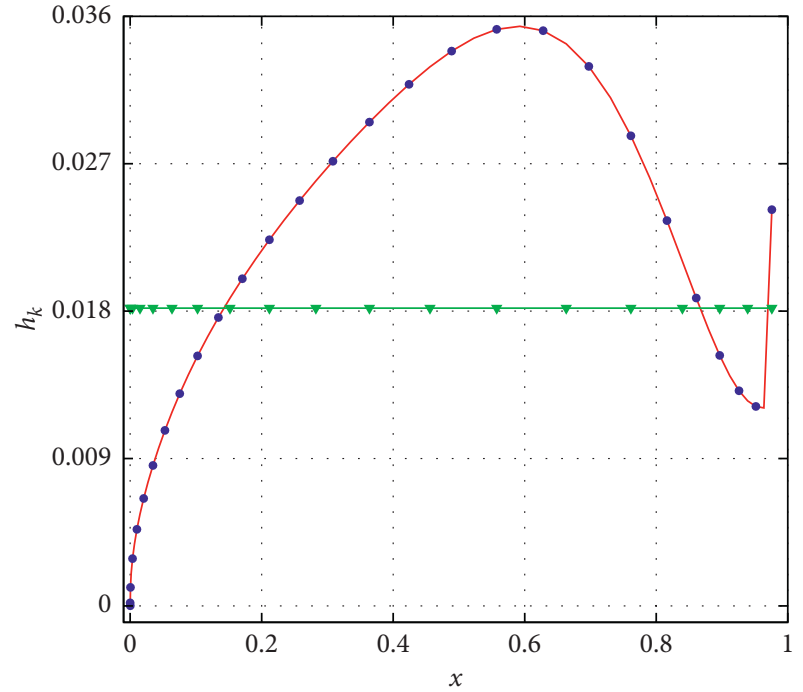

(b)

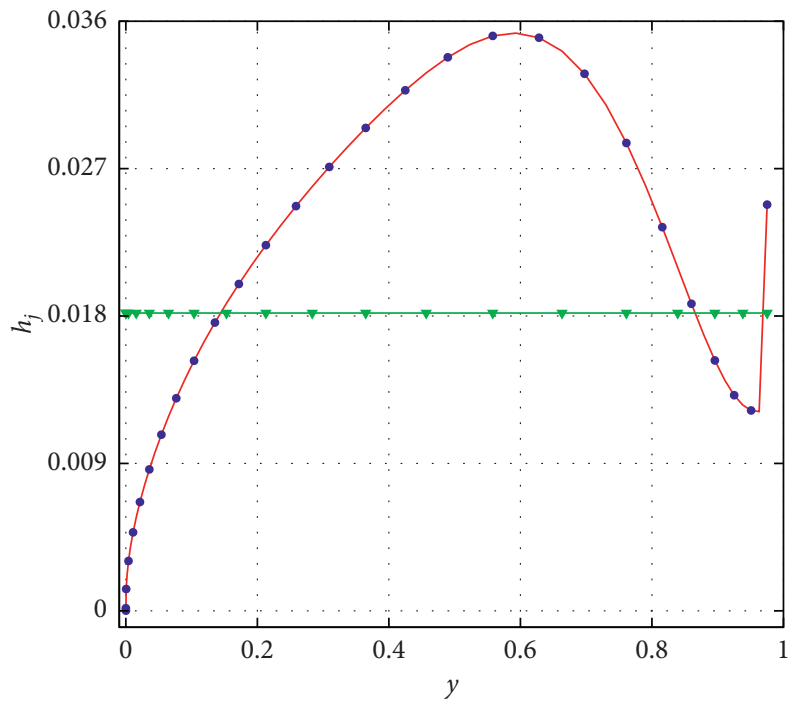

(c)

FiguRE 5: (a) The curve of the temporal steps $\tau_{n}$ as time $t$ increases, (b) the variation of spatial steps $h_{k}$ as $x$ processes, and (c) the variation of spatial steps $h_{k}$ as $y$ processes and for the convection term $b /(1+\alpha x+\beta y)$.

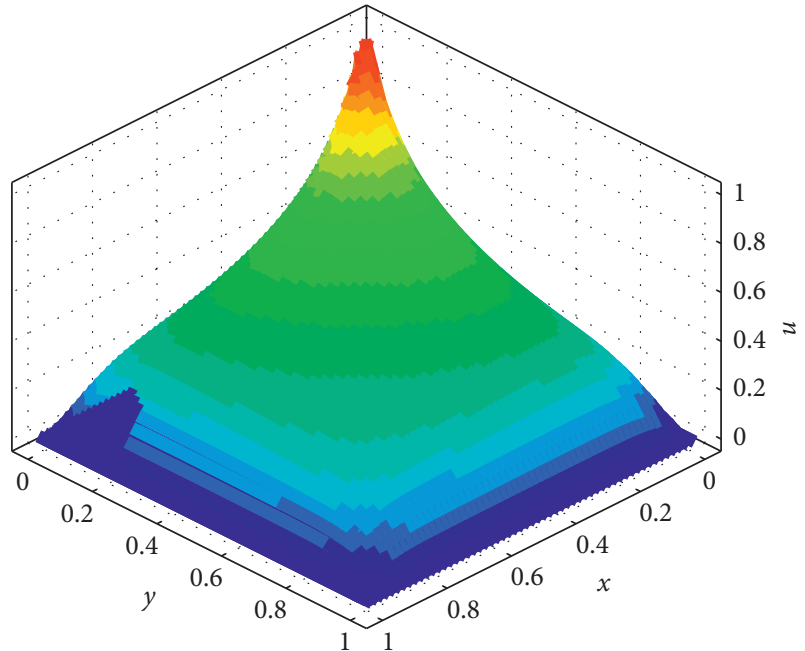

(a)

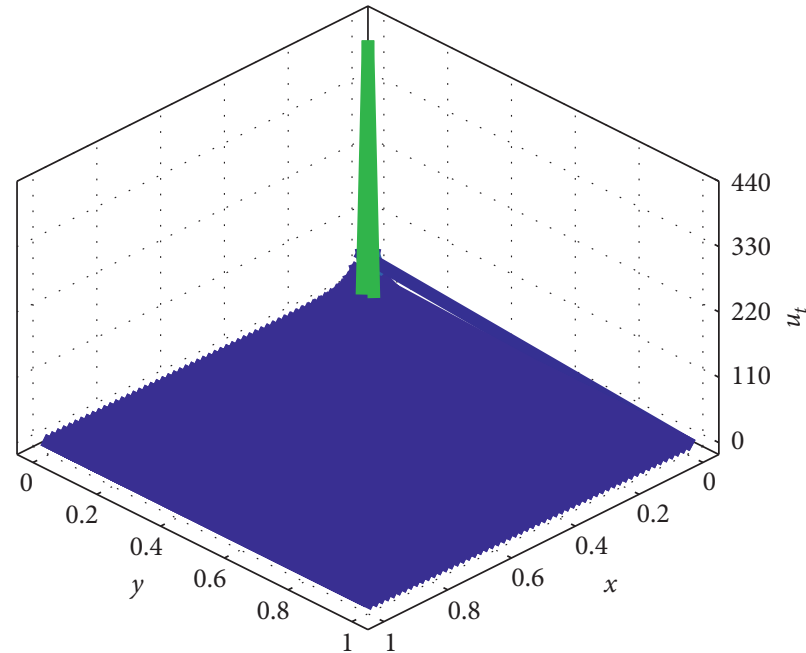

(b)

Figure 6: (a) The 3D plot of $u$ and (b) the 3D plot of $u_{t}$ when $t=4.487770702255042$ for the convection term $b /(1+\alpha x+\beta y)$. 


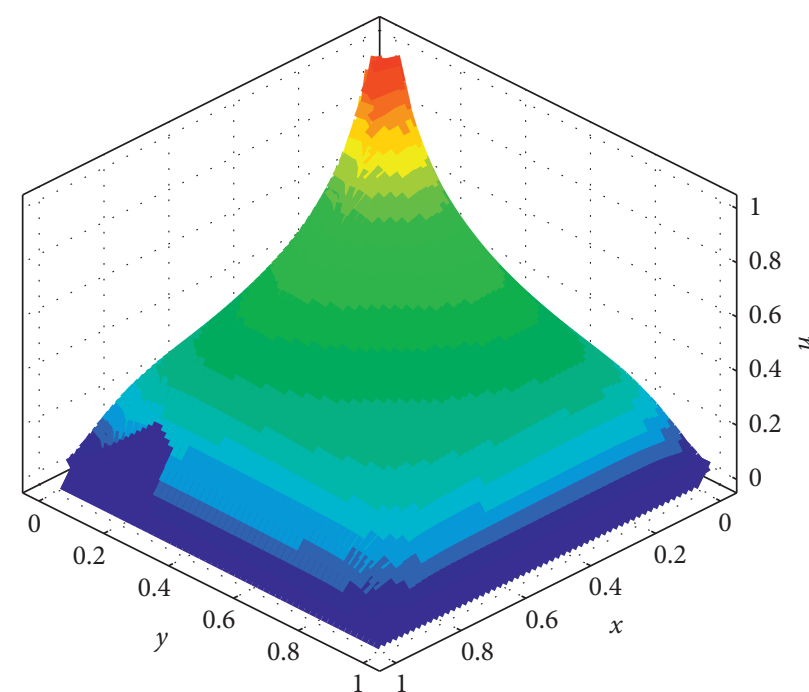

(a)

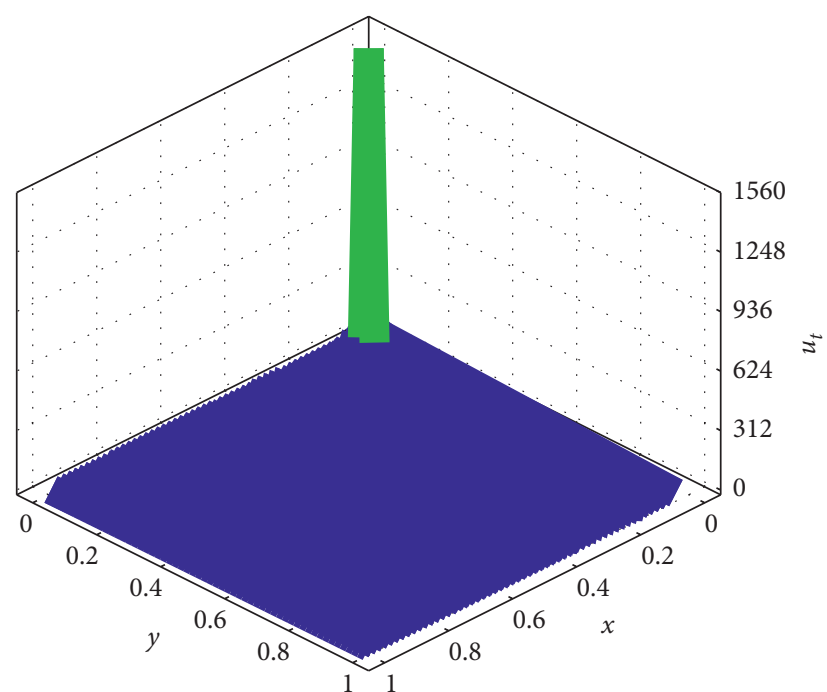

(b)

Figure 7: (a) The 3D plot of $u$ and (b) the 3D plot of $u_{t}$ when $t=T_{7}^{*}$ for the convection term $b /(1+\alpha x+\beta y)$.

TABLE 4: Time, location, $\max _{x, y} u$, and $\max _{x, y}\left(u_{t}\right)$ in the last seven temporal layers which contains quenching occurrence for Case 8.0.

\begin{tabular}{lcccc}
\hline$t$ & $x$ & $y$ & $\max _{x, y} u$ & $\max _{x, y}\left(u_{t}\right)$ \\
\hline 5.596499999999618 & 0.025 & 0.025 & 0.9656132480353845 & 27.20492580514906 \\
5.598499999999617 & 0.025 & 0.025 & 0.9668480910506297 & 28.76690218778399 \\
5.599999999999616 & 0.025 & 0.025 & 0.9678079528853171 & 30.06497816241713 \\
5.601999999999615 & 0.025 & 0.025 & 0.969139216203357 & 32.00073689849199 \\
5.603982012140827 & 0.025 & 0.025 & 0.9708363009713709 & 34.72704476646155 \\
5.604262757909247 & 0.025 & 0.025 & 0.9835341519876587 & 73.15674241637485 \\
5.604272047535358 & - & - & - & $6.637517818582074 \times 10^{11}$ \\
\hline
\end{tabular}

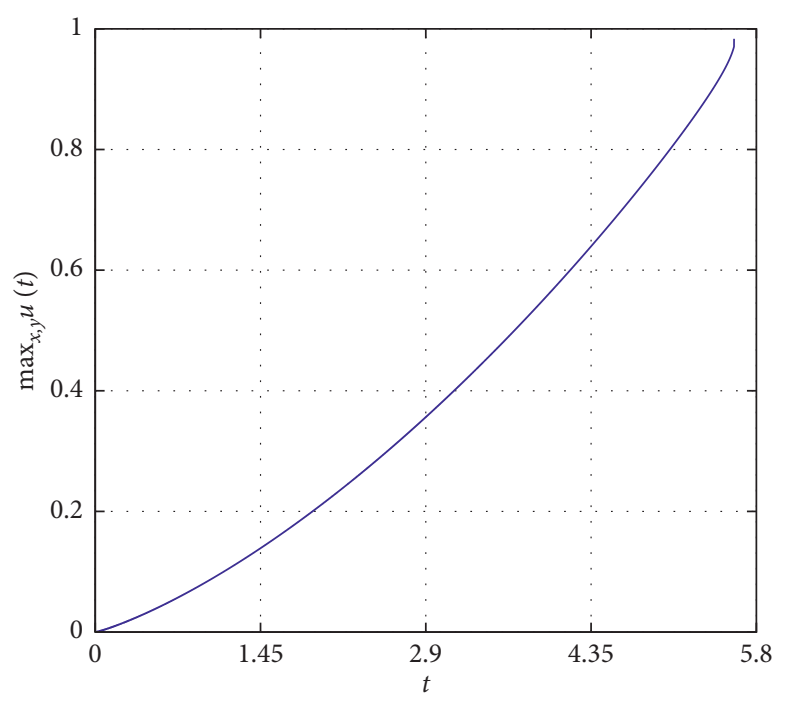

(a)

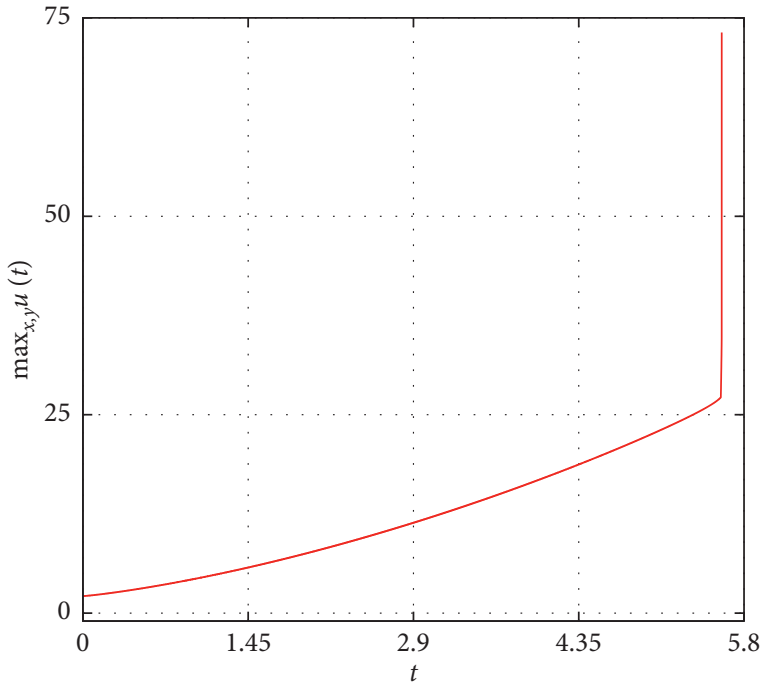

(b)

FIGURE 8: The graphs of (a) $\max _{x, y} u$ as $t$ increases and (b) $\max _{x, y}\left(u_{t}\right)$ as $t$ increases from the initial moment to the occurrence of quenching behavior for the convection term $b /(\alpha x+\beta y)$. 


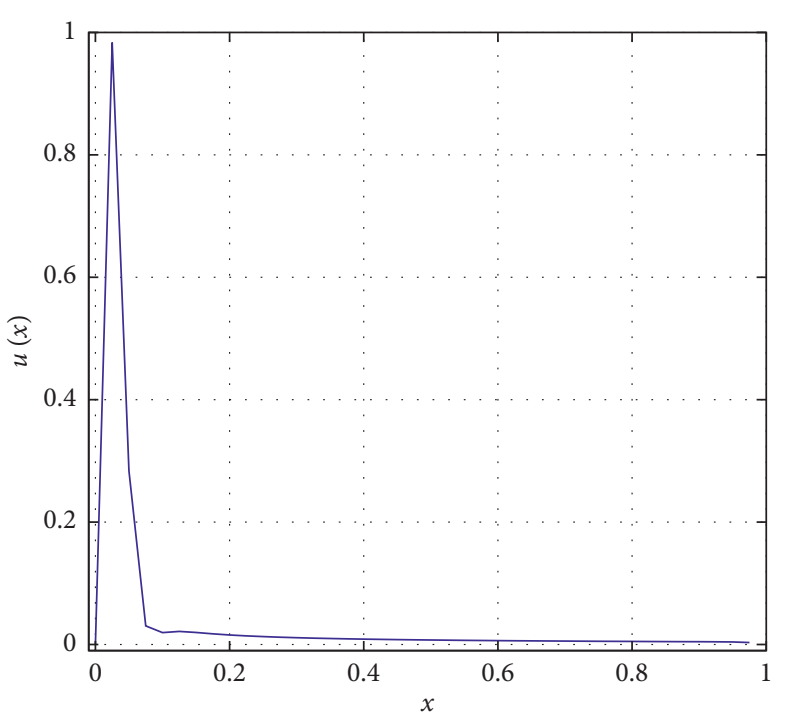

(a)

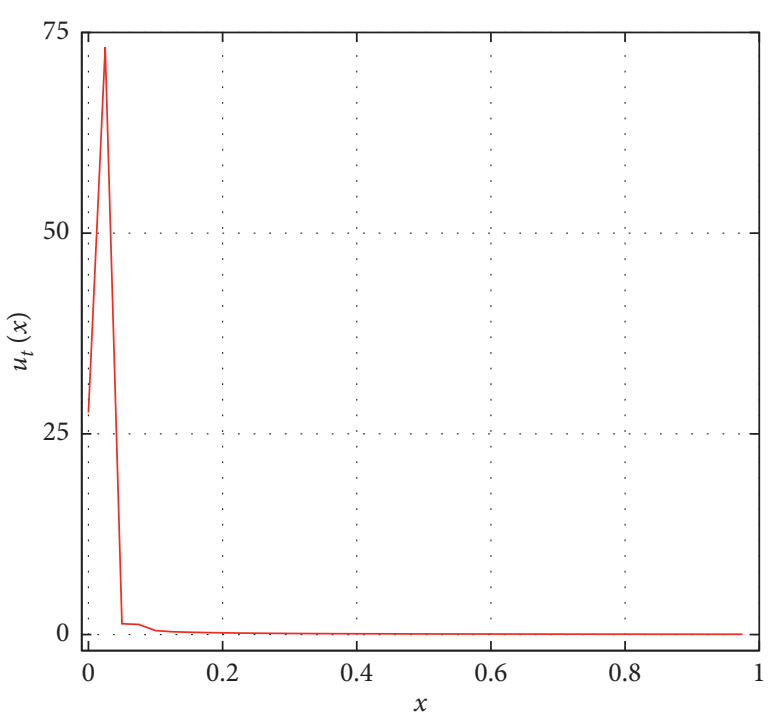

(b)

Figure 9: The graphs of (a) $u$ as $x$ increases and (b) $u_{t}$ as $x$ increases when $t$ is $T_{8}^{*}$ for the convection term $b /(\alpha x+\beta y)$.

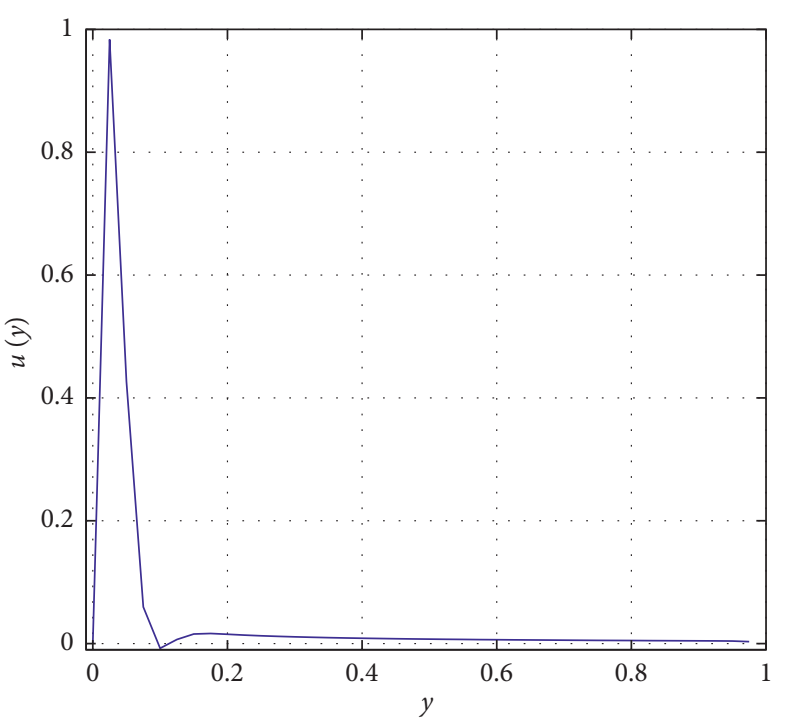

(a)

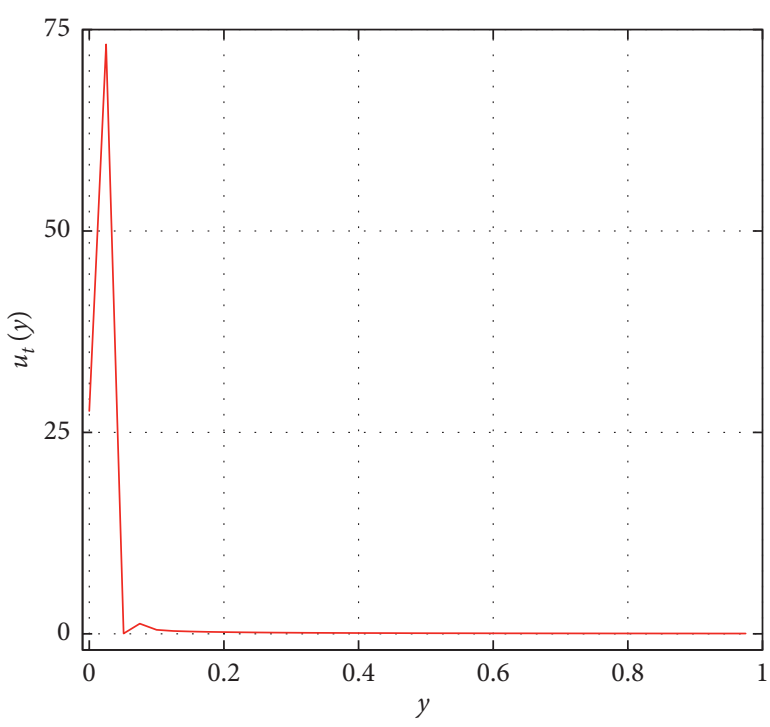

(b)

Figure 10: The graphs of (a) $u$ as $y$ increases and (b) $u_{t}$ as $y$ increases when $t$ is $T_{8}^{*}$ for the convection term $b /(\alpha x+\beta y)$.

whereas $\max _{x, y}\left(u_{t}\right)$ at other positions are positive numbers less than 1, which can be seen via Figures 9(b) and 10(b). Obviously, the larger $\max _{x, y}\left(u_{t}\right)$ are all less than 73.15674241637485 , which is reasonable for a quenching case.

4.5. The Effect of Burner Size on Quenching. The relationship between combustion the chamber shape and quenching features is discussed in this paragraph, which shows how the ratio of length to width of the burner affects the quenching behavior. In terms of $c(x, y)=b /(1+\alpha x+\beta y)$, we also consider serial values of $\alpha / \beta$ taken in $[0.7,0.97]$ besides $\alpha=\beta$ corresponding to Case 7.0 in Table 5 because it does not produce quenching behavior when $\alpha / \beta$ is smaller than 0.7 . We choose ten values: $0.7,0.72,0.82,0.85,0.88,0.89,0.9$, 0.92, 0.95, and 0.97 for Case 7.0.1-Case 7.0.10 in Table 5. Specially, Case 7.0.1: $\alpha / \beta=0.7$, Case 7.0.2: $\alpha / \beta=0.72$, Case 7.0.3: $\alpha / \beta=0.82$, Case 7.0.4: $\alpha / \beta=0.85$, Case 7.0.5: $\alpha / \beta=0.88$, Case 7.0.6: $\alpha / \beta=0.89$, Case 7.0.7: $\alpha / \beta=0.9$, Case 7.0.8: $\alpha / \beta=0.92$, Case 7.0.9: $\alpha / \beta=0.95$, and Case 7.0.10: $\alpha / \beta=0.97$. The quenching data associated with different $\alpha / \beta$ based on $q=0.5, \alpha=\sqrt{200}, b_{x}=b_{y}=10, \theta=1, \tau_{0}=0.0003$, and $h_{0}=1 / 55$ for the convection term $b /(1+\alpha x+\beta y)$ are recorded in Table 5. From Table 5, we can discover that quenching time $t_{\max }$ that is 4.5172399998669761 is the largest 


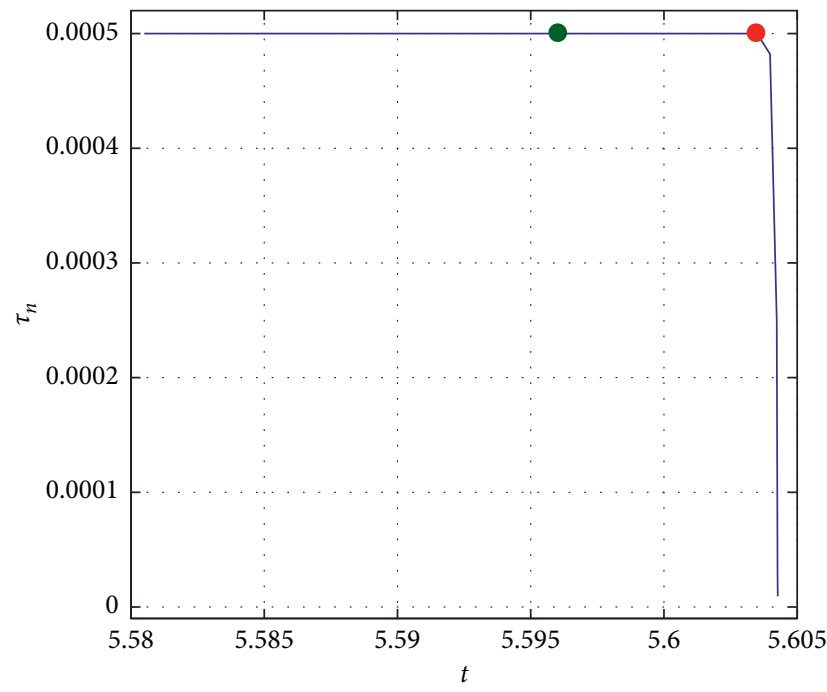

(a)

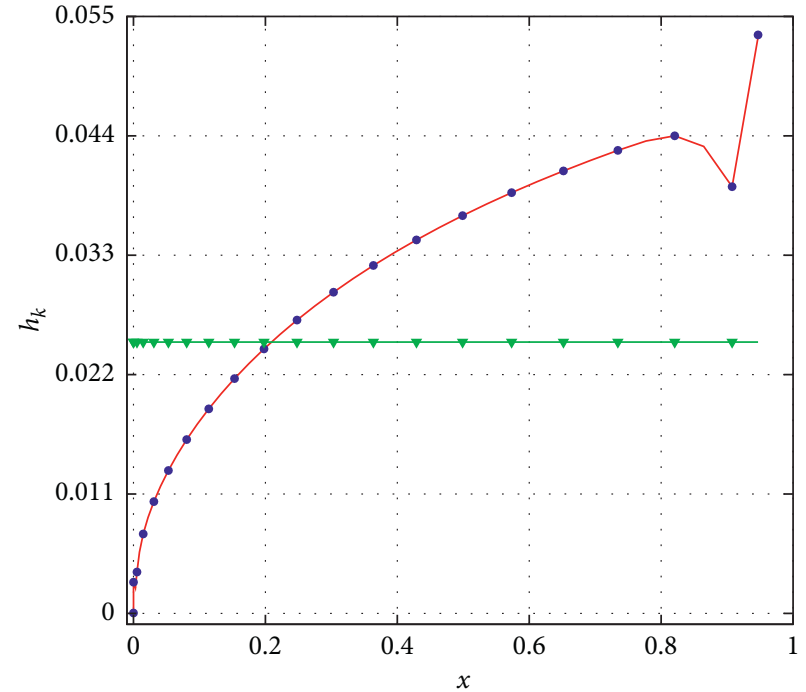

(b)

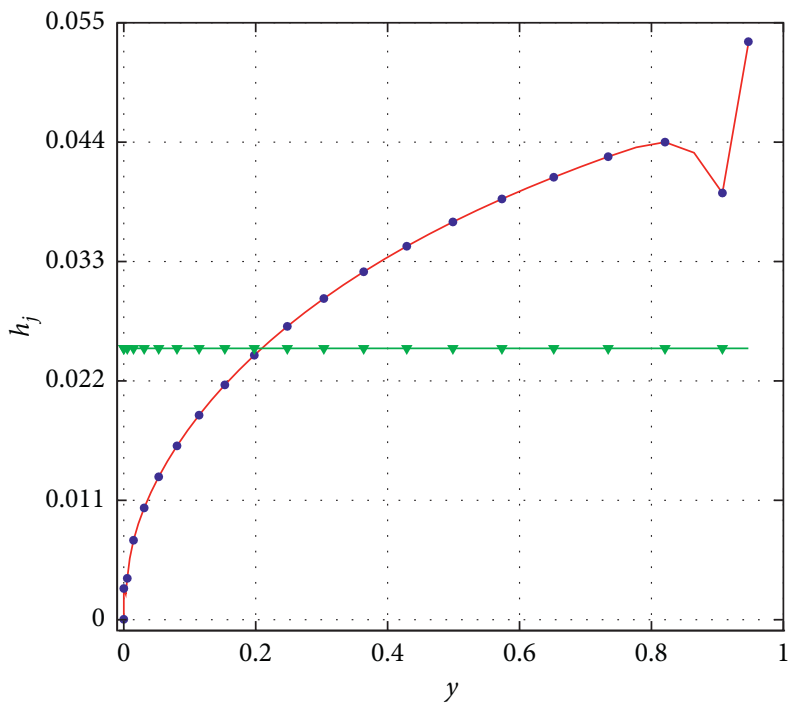

(c)

FIgURE 11: (a) The curve of the temporal steps $\tau_{n}$ as time $t$ increases, (b) the variation of spatial steps $h_{k}$ as $x$ processes, and (c) the variation of spatial steps $h_{k}$ as $y$ processes and for the convection term $b /(\alpha x+\beta y)$.

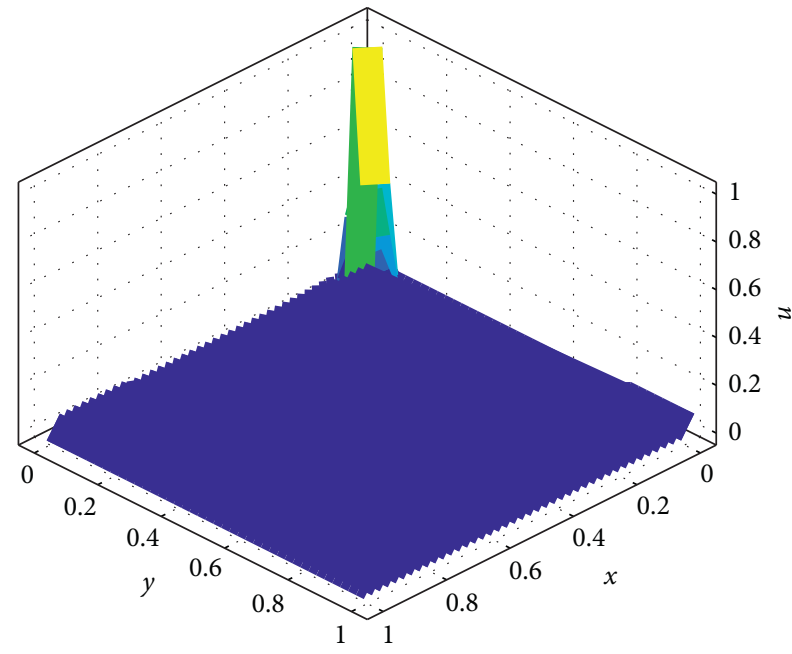

(a)

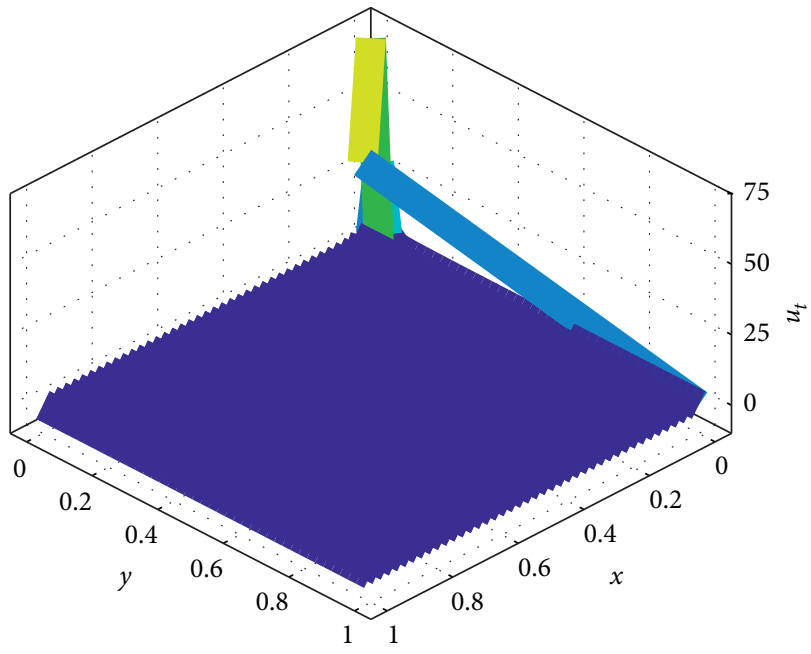

(b)

Figure 12: (a) The 3D plot of $u$ and (b) the 3D plot of $u_{t}$ when $t=T_{8}^{*}$ for the convection term $b /(\alpha x+\beta y)$. 
TABle 5: Quenching time, quenching location, $\max u$, and $\max u_{t}$ relative to different $\alpha / \beta$ when $c(x, y)=b /(1+\alpha x+\beta y)$ and $q=0.5, \alpha=\sqrt{200}, b_{x}=b_{y}=10$, and $\theta=1$.

\begin{tabular}{lccccc}
\hline Case name & $t_{\max }$ & $x_{\max }$ & $y_{\max }$ & $\max u$ & $\max u_{t}$ \\
\hline Case 7.0 & 4.487776579638927 & 0.03636363636363636 & 0.03636363636363636 & 0.9992409498317957 & 1539.153786381783 \\
Case 7.0.1 & 4.279622943667804 & 0.03636363636363636 & 0.01818181818181818 & 0.9992681723524656 & 1710.18659619492 \\
Case 7.0.2 & 4.331831171603769 & 0.03636363636363636 & 0.01818181818181818 & 0.9991903860122657 & 1552.306642881425 \\
Case 7.0.3 & 4.496584166548055 & 0.03636363636363636 & 0.01818181818181818 & 0.9991987852767854 & 1599.733801339674 \\
Case 7.0.4 & 4.517239999869761 & 0.03636363636363636 & 0.01818181818181818 & 0.9993256457315207 & 1911.903484162842 \\
Case 7.0.5 & 4.51465481210012 & 0.03636363636363636 & 0.01818181818181818 & 0.9992234514278073 & 1453.952639953373 \\
Case 7.0.6 & 4.513026286098267 & 0.03636363636363636 & 0.03636363636363636 & 0.9995938543472831 & 2793.85091710218 \\
Case 7.0.7 & 4.511208275853616 & 0.03636363636363636 & 0.03636363636363636 & 0.9997633374772214 & 4813.578815453525 \\
Case 7.0.8 & 4.50725924981429 & 0.03636363636363636 & 0.03636363636363636 & 0.9993672152218838 & 1807.556556363968 \\
Case 7.0.9 & 4.500508288781494 & 0.03636363636363636 & 0.03636363636363636 & 0.9996751433553265 & 3556.703280522448 \\
Case 7.0.10 & 4.495664373739898 & 0.03636363636363636 & 0.03636363636363636 & 0.9992396930880079 & 1524.670120016285 \\
\hline
\end{tabular}

TABle 6: Quenching time, quenching location, $\max u$, and $\max u_{t}$ relative to different $\alpha / \beta$ when $c(x, y)=b /(\alpha x+\beta y)$ and $q=1.1, \alpha=20, b_{x}=b_{y}=3$, and $\theta=1$.

\begin{tabular}{lccccc}
\hline Case name & $t_{\max }$ & $x_{\max }$ & $y_{\max }$ & $\max u$ & $\max u_{t}$ \\
\hline Case 8.0 & 5.604262757909247 & 0.025 & 0.025 & 0.9835341519876587 & 73.15674241637485 \\
Case 8.0.1 & 48.53275162233688 & 0.025 & 0.025 & 0.9800673701485662 & 11.64671094996052 \\
Case 8.0.2 & 29.17257043706389 & 0.025 & 0.025 & 0.9806176145779403 & 20.45608182578629 \\
Case 8.0.3 & 25.29371073307144 & 0.025 & 0.025 & 0.9813605780402614 & 26.01159161851154 \\
Case 8.0.4 & 24.18215821258183 & 0.025 & 0.025 & 0.9819794464122609 & 34.70682386174796 \\
Case 8.0.5 & 24.67754900263962 & 0.025 & 0.025 & 0.9810927486939868 & 39.65241020973182 \\
Case 8.0.6 & 46.46933057744767 & 0.025 & 0.025 & 0.9825870000519357 & 51.20986827535954 \\
Case 8.0.7 & 14.79035540276399 & 0.025 & 0.025 & 0.9804808252326324 & 48.88835666486023 \\
Case 8.0.8 & 15.63082052925696 & 0.025 & 0.025 & 0.9810194325889038 & 54.83303601502359 \\
Case 8.0.9 & 22.86481549877496 & 0.025 & 0.025 & 0.9806433847147185 & 56.65280512266988 \\
Case 8.0.10 & 5.160277808704819 & 0.025 & 0.025 & 0.9824769898865595 & 66.75815635770577 \\
\hline
\end{tabular}

when $\alpha / \beta=0.85$ and quenching location $x_{\max }=y_{\max }$ that is 0.03636363636363636 when $0.89 \leq \alpha / \beta \leq 1$.

Similarly, we investigate quenching behavior variation with the changes of $\alpha / \beta$ for convection function $b /(\alpha x+\beta y)$. From a number of tests, we select eleven meaningful cases to be given in Table 6. Case 8.0: $\alpha / \beta=1$, Case 8.0.1: $\alpha / \beta=0.15$, Case 8.0.2: $\alpha / \beta=0.25$, Case 8.0.3: $\alpha / \beta=0.3$, Case 8.0.4: $\alpha / \beta=0.4$, Case 8.0.5: $\alpha / \beta=0.5$, Case 8.0.6: $\alpha / \beta=0.6$, Case 8.0.7: $\alpha / \beta=0.7$, Case 8.0.8: $\alpha / \beta=0.8$, Case 8.0.9: $\alpha / \beta=0.85$, and Case 8.0.10: $\alpha / \beta=0.95$. The initial $t$-step is $\tau_{0}=0.0005$, and the initial $x$-step ( $y$-step) is $h_{0}=1 / 40 . q, \alpha, b_{x}\left(b_{y}\right)$, and $\theta$ are set as $c q=1.1, \alpha=20, b_{x}=b_{y}=3$, and $\theta=1$. It is easily seen that quenching occurs, and quenching location $x_{\max }=y_{\max }$ is 0.025 when $\alpha / \beta \geq 0.15$ and quenching time $t_{\max }$ is the smallest when $\alpha / \beta=0.375$ from Table 6 .

Figures 13 and 14 display the relationships between $t_{\max }$, $x_{\max }, y_{\max }$, and $\alpha / \beta$, respectively, in which, the red curves in Figures 13(a) and 14(a) reflect quenching time $t_{\max }$ variations as $\alpha / \beta$ rises; the blue curves in Figures 13(b) and 14(b) reflect quenching location $x_{\max }$ variations as $\alpha / \beta$ rises; the green curves in Figures 13(b) and 14(b) reflect quenching location $y_{\max }$ variations as $\alpha / \beta$ rises. Figure 13 corresponds to Table 5, and Figure 14 corresponds to Table 6 . The highest value marked by the green sign of the red contour in Figure 13(a) is the quenching time 4.517239999869761 of Case 7.0.4. Quenching time $t_{\max }$ drops monotonously as $\alpha / \beta$ increases when $0.85<\alpha / \beta \leq 1.0$, and it promotes monotonously as $\alpha / \beta$ increases when $0.7 \leq \alpha / \beta \leq 0.85$. The shape of the red contour in Figure 14(a) is different from that of the red contour in Figure 13(a). Its lowest green point is just the quenching time 5.160277808704819 of Case 8.0.10, and $\alpha / \beta$ is 0.95 . The contour shows the fluctuation of the points up and down. For the relationship between $x_{\max }$ and $\alpha / \beta$, we rely on the tendencies of the red dots on the blue curves in Figures 13(b) and 14(b), respectively, to see that $x_{\max }$ keeps a same value in each case. Depending on the trend of the purple dots on the green curves in Figures 13(b) and $14(\mathrm{~b})$, respectively, we can find that $y_{\max }$ keeps a smaller value than $x_{\max }$ when $0.7 \leq \alpha / \beta \leq 0.89$ and becomes the same value as $x_{\max }$ when $0.89 \leq \alpha / \beta \leq 1$ for Figure 13(b) and $y_{\max }$ is equal to $x_{\max }$ for Figure 14(b). Meanwhile, it is clearly observed that there is only the blue contour with ten red points extending to the end in Figure 14(b).

Finally, we can conclude that quenching problems based on different convection functions require different combustion chamber sizes. It can lead to quenching behaviors to properly take the ratio of $\alpha$ and $\beta$ when $\alpha / \beta \geq 0.7$ for convection function $b /(1+\alpha x+\beta y)$. But, it can result in quenching phenomena to properly take the ratio of $\alpha$ and $\beta$ when $\alpha / \beta \geq 0.15$ for the convection term $b /(\alpha x+\beta y)$. By comparing, it is easier to form quenching behaviors for the latter than the former. Generally, we can consider it will produce quenching phenomena when $\alpha / \beta$ takes a value not less than 0.7 . There must be a suitable ratio that will spend 


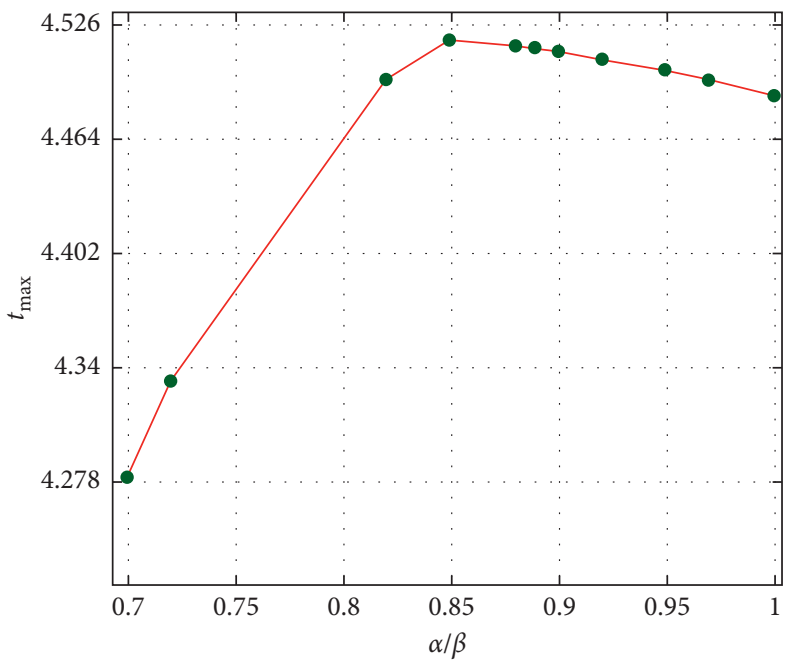

(a)

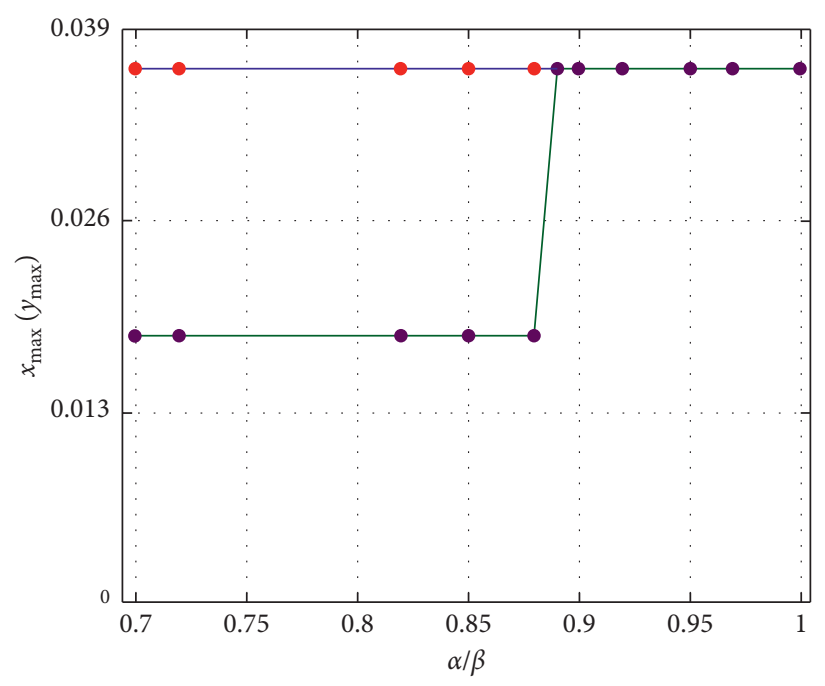

(b)

Figure 13: (a) The plot of $t_{\max }$ as $\alpha / \beta$ increases and (b) the plots of $x_{\max }$ and $y_{\max }$ as $\alpha / \beta$ increases for the convection term $b /(1+\alpha x+\beta y)$.

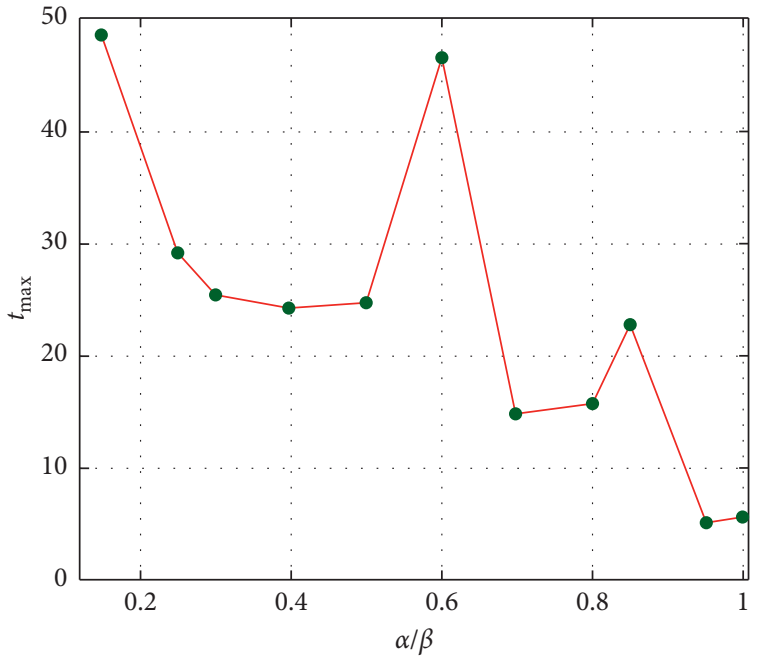

(a)

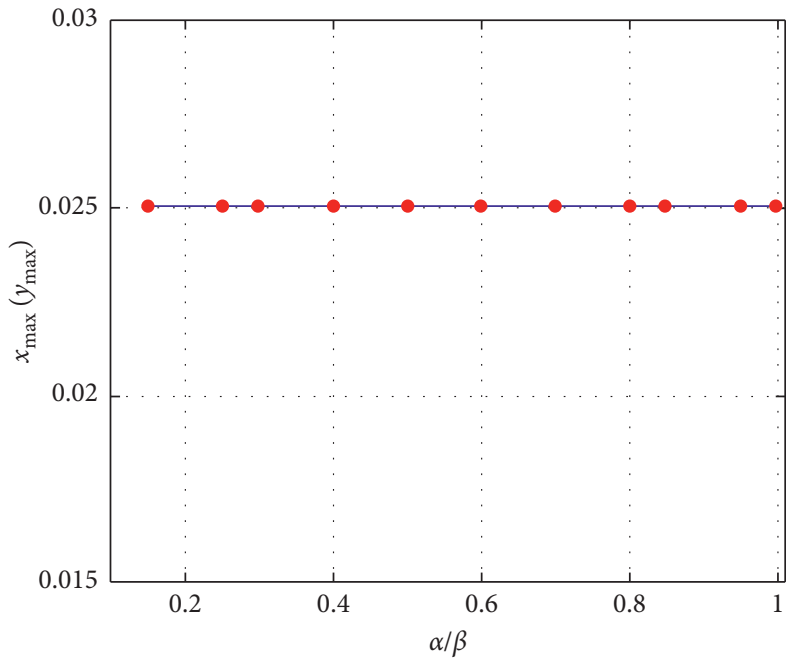

(b)

Figure 14: (a) The plot of $t_{\max }$ as $\alpha / \beta$ increases and (b) the plots of $x_{\max }$ and $y_{\max }$ as $\alpha / \beta$ increases for the convection term $b /(\alpha x+\beta y)$.

the most quenching time. When the length and the width of the combustion chamber are close (i.e., $0.89 \leq \alpha / \beta \leq 1$ ), the horizontal coordinate of quenching location is equivalent to its vertical coordinate.

\section{Conclusions}

Nonlinear singular degenerate reaction-diffusion equation plays more and more important role in the quenching area such as diffusion of thermal and energy, combustion theory, aerospace engineering, and biomedical treatment. We put forward a compact difference scheme and built an adaptation algorithm to solve the 2D convection-reaction-diffusion equation of the quenching type. Depending on theoretical and numerical experiments above, we can get some conclusions as follows. As a valid and powerful mathematical tool, the high-order compact difference scheme combining with the proper adaptive procedure may offer elegant manners to resolve the semilinear singular degenerate partial differential equation. It is very efficient to employ the two-layer self-adaptive grid principle for solving the quenching problem. The self-adaptive temporal step makes time adaptation activated when matching the critical condition. It not only saves computational cost, but also impacts on specific moment of quenching. Similarly, it is important to choose spatial adaptation parameters, which may decide where the temporal derivative will blow-up, even whether quenching occurs or not. The study provides the feasibility of analyzing and resolving different engineering applications of the quenching problem in different finite difference schemes on an adaptive mesh. We will conduct further research in the future, which includes the $3 \mathrm{D}$ 
reaction-diffusion equations with the convection function of the quenching type.

\section{Data Availability}

The data used to support the findings of this study are available from the corresponding author upon request.

\section{Conflicts of Interest}

The authors declare that they have no conflicts of interest.

\section{Acknowledgments}

This work was partially supported by the National Natural Science Foundation of China (Grant nos. 11772165 and 11961054); National Natural Science Foundation of Ningxia (Grant no. 2018AAC02003); Key Research and Development Program of Ningxia (Grant no. 2018BEE03007); and Major Innovation Projects for Building First-Class Universities in China's Western Region (Grant no. ZKZD2017009).

\section{References}

[1] H. Kawarada, On Solutions of Initial-Boundary Value Problem for $u_{t}=u_{x x}+1(1-u)$, Vol. 10, Publications of The Research Institute for Mathematical Sciences, Kyoto University, Kyoto, Japan, 1975.

[2] T. Hou, "Blow-up or no blow-up? A unified computational and analytic approach to $3 \mathrm{D}$ incompressible Euler and Navier-Stokes equations," Acta Numerica, vol. 18, pp. 277346, 2009.

[3] P. Chang and H. A. Levine, "The quenching of solutions of semi-linear hyper-bolic equations," SIAM Journal of Mathematical Analysis, vol. 12, pp. 893-903, 1982.

[4] A. Acker and B. Kawohl, "Remarks on quenching," Nonlinear Analysis: Theory, Methods \& Applications, vol. 13, no. 1, pp. 53-61, 1989.

[5] C. Chen, "The method of fundamental solutions for nonlinear thermal explosions," Communications in Numerical Methods in Engineering, vol. 11, no. 8, pp. 675-681, 1995.

[6] K. W. Liang, P. Lin, M. T. Ong, and R. C. E. Tan, "A splitting moving mesh method for reaction-diffusion equations of quenching type," Journal of Computational Physics, vol. 215, no. 2, pp. 757-777, 2006.

[7] Q. Sheng, "Adaptive decomposition finite difference methods for solving singular problems-a review," Frontiers of Mathematics in China, vol. 4, no. 4, pp. 599-626, 2009.

[8] C. Y. Chan and L. Ke, "Parabolic quenching for nonsmooth convex domains," Journal of Mathematical Analysis and Applications, vol. 186, no. 1, pp. 52-65, 1994.

[9] H. Cheng, P. Lin, Q. Sheng, and R. C. E. Tan, "Solving degenerate reaction-diffusion equations via variable step peaceman-rachford splitting," SIAM Journal on Scientific Computing, vol. 25, no. 4, pp. 1273-1292, 2003.

[10] M. A. Beauregard and Q. Sheng, "An adaptive splitting approach for the quenching solution of reaction-diffusion equations over non-uniform grids," Journal of Computational and Applied Mathematics, vol. 241, pp. 30-44, 2013.

[11] J. L. Padgett and Q. Sheng, "Non uniform crank-nicolson scheme for solving the stochastic Kawarada equation via arbitrary grids," Numerical Methods for Partial Differential Equations, vol. 33, no. 4, pp. 1305-1328, 2017.

[12] A. Q. M. Khaliq and Q. Sheng, "On the monotonicity of an adaptive splitting scheme for two-dimensional singular reaction-diffusion," International Journal of Computer Mathematics, vol. 84, no. 6, pp. 795-806, 2007.

[13] M. A. Beauregard and Q. Sheng, "A semi-adaptive compact splitting method for the numerical solution of 2-dimensional quenching problems," Applied Mathematics and Computation, vol. 218, no. 22, pp. 11240-11254, 2012.

[14] M. A. Beauregard and Q. Sheng, "Solving degenerate quenching-combustion equations by an adaptive splitting method on evolving grids," Computers and Structures, vol. 122, pp. 33-43, 2013.

[15] M. A. Beauregard, "Numerical solutions to singular reaction diffusion equation over elliptical domains," Applied Mathematics and Computation, vol. 254, pp. 75-91, 2015.

[16] J. L. Padgett and Q. Sheng, "Numerical solution of degenerate stochastic Kawarada equations via a semi-discretized approach," Applied Mathematics and Computation, vol. 325, pp. 210-226, 2018.

[17] J. L. Padgett and Q. Sheng, "On the stability of a variable step exponential splitting method for solving multidimensional quenching-combustion equations,"in Modern MathematicalMethods and High Performance Computing in Science and Technology, Springer Proceedings in Mathematics \& Statistics, vol. 171, pp. 155-167, Springer, Berlin, Germany, 2016.

[18] Y. Ge, Z. Cai, and Q. Sheng, "A compact adaptive approach for degenerate singular reaction -diffusion equations," $\mathrm{Nu}$ merical Methods for Partial Differential Equations, vol. 34, no. 4, pp. 1166-1187, 2018.

[19] Q. Sheng and A. Q. M. Khaliq, "A compound adaptive approach to degenerate nonlinear quenching problems," $\mathrm{Nu}$ merical Methods for Partial Differential Equations, vol. 15, no. 1, pp. 29-47, 1999.

[20] Q. Sheng and H. Cheng, "An adaptive grid method for degenerate semi-linear quenching problems," Computers and Mathematics with Applications, vol. 39, no. 9-10, pp. 57-71, 2000.

[21] Q. Sheng and A. Q. M. Khliq, "Adaptive algorithms of convection-diffusion-reaction equations of quenching type, dynamic continuous," Dynamics of Continuous, Discrete and Impulsive Systems Series A: Mathematical and Analysis, vol. 8, pp. 129-148, 2001.

[22] Q. Zhou, Y. Nie, X. Zhou, and W. Guo, "Quenching of a semilinear diffusion equation with convection and reaction," Electronic Journal of Differential Equations, vol. 2015, no. 1, pp. 1-7, 2015.

[23] X. Zhu and R. Hong, "High-order compact difference scheme of $1 \mathrm{D}$ nonlinear degenerate convection- reaction-diffusion equation with adaptive algorithm," Numerical Heat Transfer, Part B: Fundamentals, vol. 75, no. 1, pp. 43-66, 2019.

[24] P. Sun, L. Chen, and J. Xu, "Numerical studies of adaptive finite element methods for two dimensional convectiondominated problems," Journal of Scientific Computing, vol. 43, no. 1, pp. 24-43, 2010.

[25] V. Joshi and R. Jaiman, "A positivity preserving variational method for multi-dimensional convection-diffusion-reaction equation," Journal of Computational Physics, vol. 339, pp. 247-284, 2017.

[26] J. C. Kalita, A. K. Dass, and D. C. Dalal, "A transformationfree HOC scheme for steady convection-diffusion on nonuniform grids," International Journal for Numerical Methods in Fluids, vol. 44, pp. 33-53, 2004. 
[27] Y. Ge and F. Cao, "Multigrid method based on the transformation-free HOC scheme on nonuniform grids for $2 \mathrm{D}$ convection diffusion problems," Journal of Computational Physics, vol. 230, no. 10, pp. 4051-4070, 2011.

[28] X. Huang and Y. Ge, A High-Order Compact ADI Method on Non-uniform Grid for Unsteady Convection-Diffusion Equations, Ningxia University, Yinchuan, China, 2013, in Chinese.

[29] L. Li, Z. Jiang, and Z. Yin, "Fourth-order compact finite difference method for solving two-dimensional convection-diffusion equation," Advances in Differential Equations, vol. 234, pp. 1-24, 2018.

[30] K. M. Owolabi, "Mathematical analysis and numerical simulation of patterns in fractional and classical reaction-diffusion systems," Chaos, Solitons and Fractals, vol. 93, pp. 89-98, 2016.

[31] E. Pindza and K. M. Owolabi, "Fourier spectral method for higher order space fractional reaction-diffusion equations," Communications in Nonlinear Science and Numerical Simulation, vol. 40, pp. 112-128, 2016.

[32] J. Li, Y. Qiao, S. Zhai, and X. Feng, "Meshless local Petrov Galerkin method for 2D 3D nonlinear convection diffusion equations based on LS RBF PUM," Advances in Differential Equations, vol. 74, no. 1, pp. 450-464, 2018.

[33] K. M. Owolabi and A. Atangana, "Numerical simulation of noninteger order system in subdiffusive, diffusive, and superdiffusive scenarios," Journal Computational and Nonlinear Dynamics, vol. 12, no. 3, 2017. 